\section{HILOS DE TEATRO: LA PUESTA EN ESCENA DEL PALACIO DE LOS}

\section{SOVIETS DE LE CORBUSIER, 1931}

STRANDS OF THEATRE: LE CORBUSIER'S STAGING OF THE PALACE OF THE SOVIETS, 1931 Josefina González Cubero

RESUMEN El proyecto para el concurso del Palacio de los Soviets en Moscú (1931) de Le Corbusier plantea otra posible y más fragmentaria clave de interpretacion que, aunque en principio sea mass marginal, merece ser mencionada: I la relacion con el teatro, con la renovacioion de la escena europea y con la experimentación sovieticica, porque Le Corbusier es consciente de que la arquitectula luz o directores de escena como Max Reinhardt, con el espectáculo de masas, y Vevelodod Meyerhold, con el teamo Appia, con Kabuki, van penetrando en el sentir de un Le Corbusier que deja la impronta teatral en la puesta en escena de la megaestructura PALABRAS CLAVE Le Corbusier, Concurso, Palacio de los Soviets, Teatro, Reinhardt, Meyerhold.

SUMMARY Le Corbusier's design submitted to the public competition for the Palace of the Soviets in Moscow (1931) may have ship between theatre and the resetting of the stage in Europe and Soviet experimentational, Le Corbusier was aware that architecture goes beyond the building itself and is also a sort of staging or dramaturgy. Set designers such as Appia, through the use of light, or stage directors like Max Reinhardt, through the use of performance for the masses, and Vsevelod Meyerhold, through Japonese PALABRAS CLAVE Le Corbusier, Competition, Palace of the Soviets, Theatre, Reinhardt, Meverhold.

Persona de contacto / Corresponding author: josefina.gonzalez.cubero@tap.uva.es. Escuela Técnica Superior de Arguitectura. Universidad de Valladoliid.

1. Hans Poelzig. Planta de situación previa al futuro Falacio de los Soviets con la lolesia del Cristo Salva

dor (1832), Moscú.

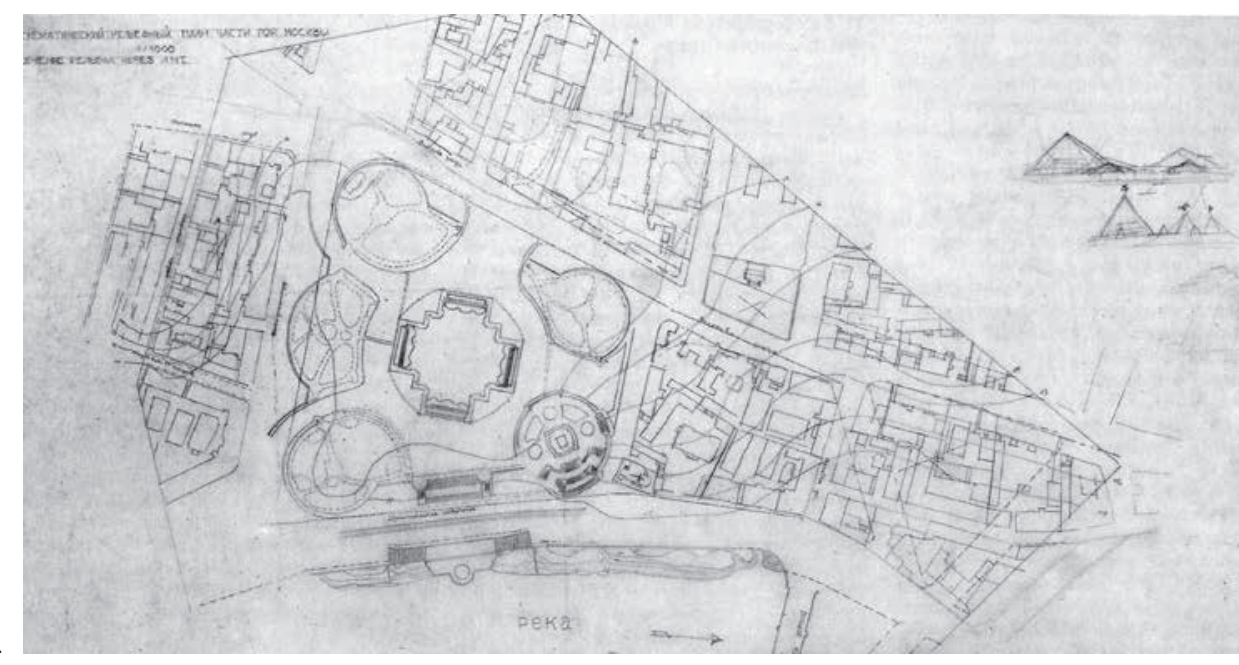

CONCURSO Y PROYECTO

pesar de la derrota de Le Corbusier en el conS beligerante ante elf fallo del concurso, contenida en ellibro Una casa un palacio (1928), consagra su proyección internacional Converido en profeta de la nueva arquitectura y del urbanismo, acomete una intensa actividad viajera a ráz de las invitacions que recibe para impartir conferencies y diseñar proyectos, lo cual le ayuda a estrechar los vínculos con la vanguardia artística internacional En este tiempo sus contactos con la Unión Sovidtca están consolidados, la obra del Centrosoyuz en marcha, ha realizado varias propuestas sobre el urbanismo de Moscá y se le reconoce a través de la traducción de sus publicaciones. Sin embargo, la entusiasta acogida de su primer viaje al país se ha transformado en una critica frecuente a su formalismo, influida por el sector alemán dentro de los CIAM'. Es a lavuelta de su curto paso por España, cuando recibe el 2 de septiembre de 1931 la invitación a pa

para el Palacio de los Soviets de Moscú como encargo remunerado

En sintoń con ba as racon decimonoca de con-

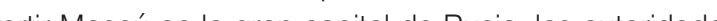
bolcheriques prosiguen la labor usta, las au ond cu victoria inciada con el concurso para et Palacio del Tabajo (1922), de ver cumplich esta vieja aspiación. La nueva convcatoria del concurso, creada bajo la sign-ficativa denominacín de Palacio de los Sovies, prende conseguir una mayor proyección del mismo, dentroy fuera de la URSS, po lo aue se promueve sin escatima

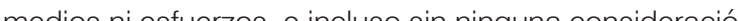
hacia lacindad existente. El emplazamiento elegido para el proyecto es un área adyacente al Kremlin junto al borde delí, lo que implica el deribo de biglesia del Cristo Salvador construida en 1832 y demolida con gran rapidez para este fin (figura 1). ante ambiguas: debe ser nificado como emblema

1. Borngräber, Christian: "Le Corbusier a Moscc"."En Rasseǵna, "Clienti i Le Corbusier", № 3, marzo 1980, Milano, pp. 79-88. 2. De Feo, Vitorio: La a arquitectura en la URSS 1917-1936 (1963). Madrid: Alianza, 1979, p. 85. Cohen, Jean-Louis. Le Corbusier et la mystique de IURSS. Bruxelles-Liège: Pierre Mardaga, 1987, pp. 205-206. 


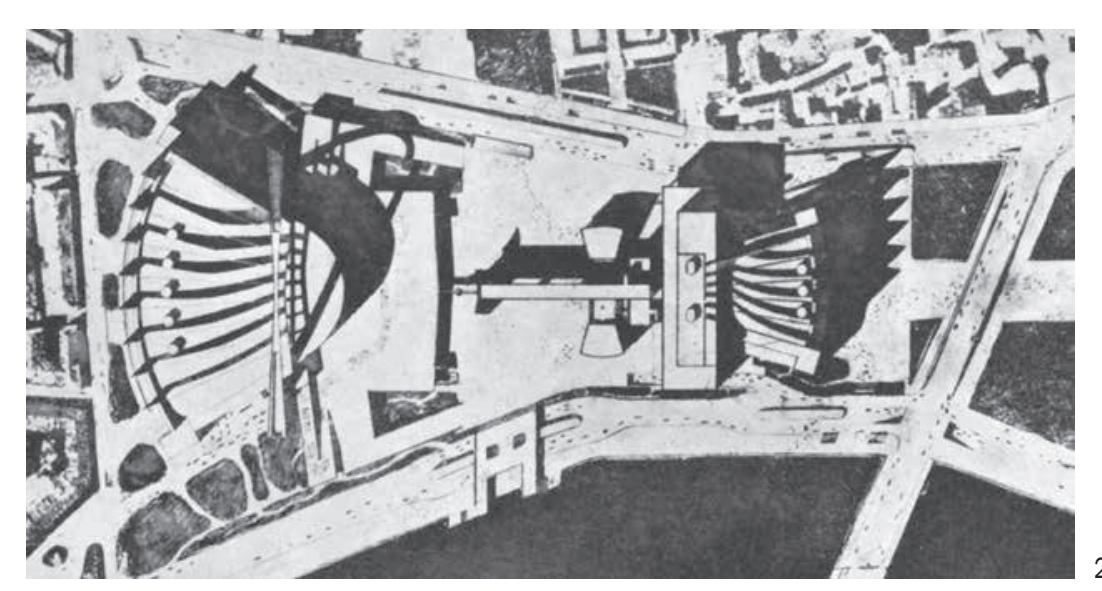
y minuciosas. En cuanto al programa, por un lado, se exi-

Si bien las características arquitectónicas están bas- el contrario, las urbanísticas y de programa son precisas ge la ordenación urbanística para permitir la concurrencia y movimiento de 25.000 personas al mismo tiempo; por otro, se trata de un programa de dimensiones notables: dos grandes salas, la mayor de 15.000 espectadores y la segunda, destinada para congresos pero sin olvidar los espectáculos, de casi 6.000 plazas. A ellas se añaden cuatro salas de conferencias para las subdivisiones de los congresos y actividades cotidianas, dos de 500 plazas y otras dos de 200 plazas cada una, y un cuarto grupo de dependencias de equipamiento y administrativas. tante desdibujadas, algo más se precisa sobre las salas respecto a las condiciones acústicas y de equipamiento tecnologico. No obstante, se detallan las actividades que debe acoger la gran sala: "El diseño de la sala debe satisfacer las necesidades de las reuniones masivas y debe resolver la cuestion de su uso para las representaciones leatrales o cinematograticas de masas, para la presentación de invenciones técnicas e industriales y también para diversas formas de participación de los espectadores en la sala, que necesitan tener acceso al escenario (al estrado o a la arena)". La confusión de la que parten las bases es significativa, pues se demanda una sala desmesurada

que pueda acoger todo tipo de espectáculos, incluso que las grandes manifestaciones puedan atravesarla $\mathrm{No}$ hay precedentes para acoger esta amalgama de usos $y$ el concurso se convierte en el reto de cómo concebir un nmenso espacio que permita cualquier representación para una congregación de espectadores multitudinaria. Se decide acometer el concurso en varias fases ${ }^{4}$ Una fase preliminar se lleva a cabo en forma de consulta interna entre los arquitectos soviéticos para elaborar las bases del concurso. A continuación, el lanzamiento de la consulta internacional es otra oportunidad para medirse las diversas lineas existentes de la arquitectura, aceptan la invitación Le Corbusier, Walter Gropius, Erich Mendelsohn, Auguste Perret, Hans Poelzig, etc., entre los numerosos concurrentes. El 28 de febrero de 1932 se hacen públicos los resultados que dejan desierto el primer premio y el encargo de la construcción. Sin embargo, se conceden dos premios destacados al arquitecto tradicionalista Ivan Znoltovsky y al americano Hector Hamilton Descarlados los grandes nombres de la esfera internacional de la invilación a participar en la segunda fase, e concurso pierde el relievey alcance de la anterior, ante e econocimiento que obtienen los proyectos académicos en las siguientes fases ${ }^{5}$ l la postergacion del IV CIAM por las autoridades

3. "La disposition de la salle doit répondre aux besoins des rassemblements des masses et doit résoudre la question de son utilisation pour des représente-

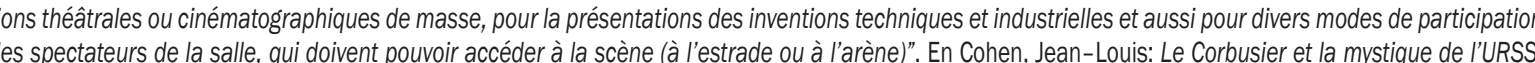
Op. cit, p. 206 .

4. Las consultas que se realizaron fueron cuatro: FASE PRELIMINAR. Entrega: febrero de 1931. Participantes: 15 proyectos. Resultados: Sin premios (consulta). $1^{\mathrm{F}}$ FASE. Comienzo: 18 de julio- Entrega: 20 de octubre de 1931 ( pospuesta hasta 1 de diciembre). Participantes: 272 proyectos ( 160 completos y 112 parcia

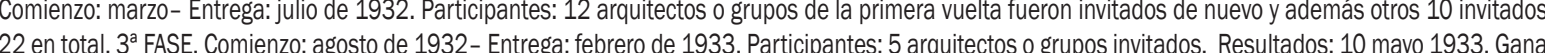

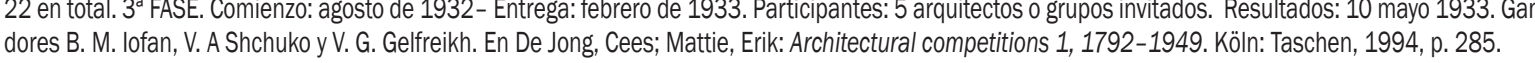

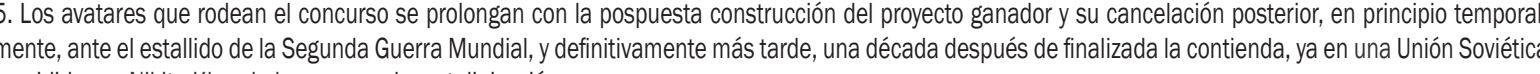

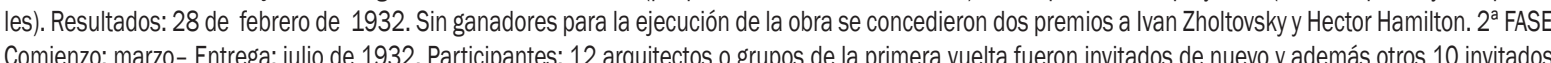
presidida por Nikita Khrushchev con su des-stalinización.
El proyecto para el Palacio de los Soviets de Le Corbusiery Pierre Jeanneret ha sido objeto de interesantisimos estudios ${ }^{6}$, que lo han analizado en las fases de desarrollo y en su resultado, con la consideración unánime de su singularidad. Es por ello que no se pretende volver soson exclusivas del proyecto, ni dentro de la obra de sus autores, ni en relación a las otras propuestas presentadas al concurso; son deudoras del solar, el programa y las bases del concurso. La forma del solar, asimilable a un estrecho trapecio tumbado, con el dominante programa bipolar de las dos grandes salas, la escala de la intervención y el uso por grandes aglomeraciones humanas les conduce, como a orros arquitectos, hacia el uso de la simetria, la aulonomia respecto del entonoy la inevitable utilización de superficies curvas', las tres características

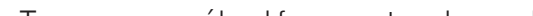
a la manera del Palacio de la SDN, el Centrosoyuz o la los be Runge, linando se ha reducido la presencia de delimitaros espacias de las fomas singuar que foriaban cón por partes de Capitel" ni el denominado espacio convexo de Lucan ${ }^{11}$ son caracteŕsticas exclusivas de bre lo dicho, sino añadir nuevas lineas de interpretación pues muchas de las caracteristicas que se le atribuyen no

Tampoco es solo tragnento urbano de Colquhoun 9 proyecto, otras propuestas las dejan traslucir bajo el ropaje de los estilos. De todas estas líneas de interpretación participa el proyecto, pero ninguna es monopolio de su propuesta.

La diferencia de las salas del Palacio de los Soviets respecto a la del Palacio de la SDN de Ginebra, compacta y cerrada, es su esponjosidad o espacialidad exterio su carácter centrífugo en planta y, más aún, en sección por la expresiva estructura resistente, lo que le contieren que parece exhibir más que en cualquier otro proyecto. sin renunciar a su constitución orgánica'2 ${ }^{2}$, la que anima investigar por otras vías

Le Corbusier presenta el proyecto más constructivista entre todos los participantes (figura 2). Se han indicado multiples influencias en su configuración. estructuras orgánicas, ingeniería de las célebres obras de Eugene polítices, etc Aun as hay que señalor otras pequeñ poincin lir de una conexión diferente, aqu se plantea ots a posib-

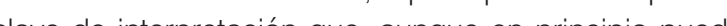
cer más marginal merece ser mencionada pa peción con el teatro, con la renovación de la escena europea y en particular con la experinentación sovielica, pora una apariencia más de artefacto que de edificio. Y es esta condiciôn de construcción del conjunto arquitectónico.

6. Destacan el de Jean-Louis Cohen por ser el más exhaustivo, también los de Stanislaus von Moos, Allen Brooks, Kenneth Frampton y Jacques Lucan, 0 trabajos más acotados como los de Alan Colluhoun y Antón Capitele entre otros.

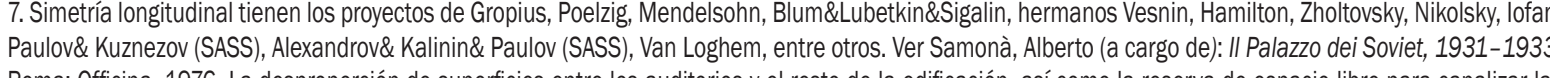
Roma: Aficina, masas de espectadores, es tan grande que los proyectos no pueden establecer conexión con la escala y forma de la ciudad. Por otro lado, el uso de las super

3.Segén $F$ ring 72

9. Colquhoun, Alan: "Las estrategias de los Grands Travaux". En Modernidad y tradición clásica (1989). Madrid: Júcar, 1991, pp. 155-198. 10. Capitel, Antón: La arquitectura compuesta por partes. Barcelona: Gustavo Gili, 2009.

11. Lucan, Jacques: "Lespace convexe: Le Corbusier et le plan libre". En Lucan, Jacques: Composition non-composition. Architecture et théorries, XXXe-XXe siècles. Vol. 1. Lausanne: Presses polytechniques et universtalies romandes (PPUR), 2009.

12. Le Corbusier et Pierre Jeanneret. OEuvre complète 1929-34 (1934). Zurich: Les Editions d'Architecture, 1995, p. 135.

13. Von Moos emparenta el proyecto con un inmenso reloj de arena, con las estructuras orgánicas o las realizaciones de Freyssinet. Von Moos, Stanislaus: Le

14. Quetglas establece el origen del arco parabólico del Palacio de los Soviets en el collar del tiro de enganche ruso de los animales de tracción a los carros.

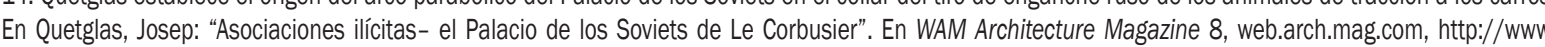
arranz.net/web.arch-mag..com/8/homeless/08s.hthm. Tambien en Quetglas, Josep: "Eyes which do not see. 4: Palace of the Soviets". En Massilia: anuario de estudios lecorbusierianos, 2002, pp. 120-122; y en Quetglas, Josep: "0jos que no ven. 4 : los caballos". En Massilia: anuario de estudios lecorbusierianos.
Encuuentro de Granada, 2008, pp. 281-285. 
trisentation: de Hamles?

inpte' havis, he moindres i $4 \mathrm{~m}$.

Le Timater Reaten far

littmamn. fait ass bir.

iutinim. Linterien. etoms

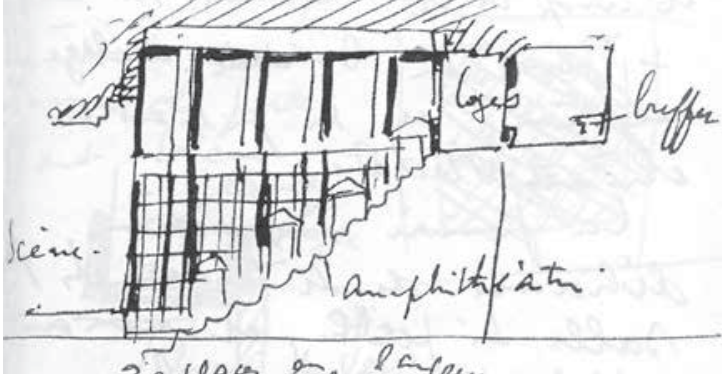

30 place as $x$ layem

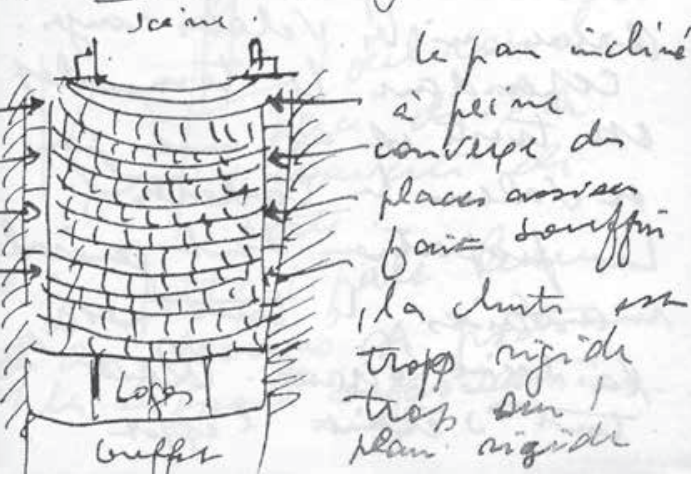

Le Corbusier es consciente de que la arquitectura no es sólo la cualidad de un edificio, es también una puesta en escena, una dramaturgla que admira tanto en la Piazza dei Miracoll de Pisa, cuyo croquis situa junto al del Palacio de los Soviets's, como en la Acropolis de Atenas: "El plan es concebido para una visión lejana. los ejes siguen el valle y las falsas escuadras son las habilidades del gran

PRIMER FRAGMENTO: CH.-É. JEANNERET, MAX REINHARDT Y LA ESCENA GERMÁNICA

Hay que retrotraerse a los viajes que Le Corbusier rea-

3. Le Corbusier (Ch.-E. Jeannerett). Apun nich, carnet 3,27 de octubre de 1910 .

del cabaret vienés El Murciélago -Fleder-

maus- (1907) de Josef Hoffmann en un

LEplattenier, s.a

Jeanneret, para encontrar la confirmación del interés por el mundo del teatro en toda su amplitud, al mismo tiempo que aprende las nuevas tecnicas de la construcción. Su estancia para estudiar las artes aplicadas industriales del pais es tambien un periodo de intenso conocimiento de la capital, asi como de otras imporlantes ciudades alemanas que visita, mientras recopila información para su futura obra La consiruction des villes que quedará inconclusa. Uno de los temas que le interesan y son objeto de estudio, reflejado en sus anotaciones y dibujos, es la arquilectura de los audilorios, teatros, salas de conciertos y, ¿como no?, sus espectáculos.

Es un momento de efervescencia cultural en la escena germánica que invita a acudir tanto a los espectáculos Iradicionales como a los nuevos e, indudablemente, a conocer el marco fisico en el que se desenvuelven. En me dio de este clima, el joven Jeanneret asiste a conciertos, operas y obras de tearro que le brindan la oportunidad de conocer de cerca las arquitecturas y criticar con veheEn

Entre las representaciones a las que acude durante (9 de la opera Tistan e Isolda de Wagner, compositor por e que siente una especial predilección, en mayo salva re sus vituperios Elekrra de Strauss, con hibreto de Horampoce lampoco sale bien parado el Hanlet que contempla e Thear- Max Litta el Teatro de los Aristas -Kunsller Theater. Max Littman acabe de los Arus as - Kunsher asesor de Georg Fuchspara la exposicion de Antesy forma significativa del espacio teatrat Los decoracos de

15. Croquis del 4 de junio de 1934. En Le Corbusier et PPierre Jeanneret. OEurre complète 1929-34 (1934). Op. citt, p. 132

16. Le Corbusier: Hacia una arquitectura (Vers une architecture, 1923). Barcelona: Poseidón, 1978, p. 39.

17. Le Corbusier es un melómano por tradición familiar, su madre era professora de música y pianista, profesión que adopta su hermano Albert, y pronto le inculca el gusto por los conciertos y óperas que frecuenta con gran asiduidad durante su estancia en Viena, de forma más espaciada en París y habitualmente en diversas ciudades de Alemania.

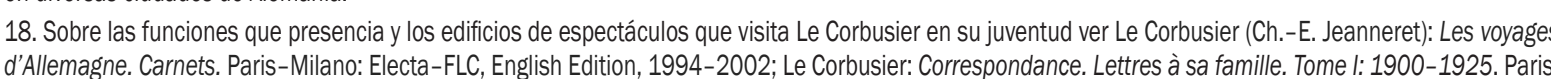

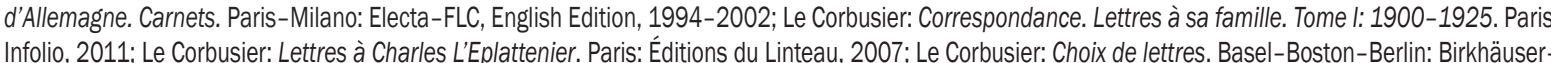
Editions d'Arrchitecture, 2002; De Simone, Rosario: Ch. E. Jeannerete-Le Corbusier Viaggio in Germania 1910-1911. Roma: Offizina Edizioni, 1989; Damish, Hubert: “Modernité: Les tréteaux de la vie moderne", en Lucan, Jacques (Ed.): Le Corbusier, une encyclopédie. Paris: Centre Georges Pompidou, 1987, pp.
252-259. Tambiein en la tesis doctoral publicada Quesada, Fernando: La caja mágica. Cuerpo y escena. Barcelona: Fundación Caja de Arquitectos, 2005.

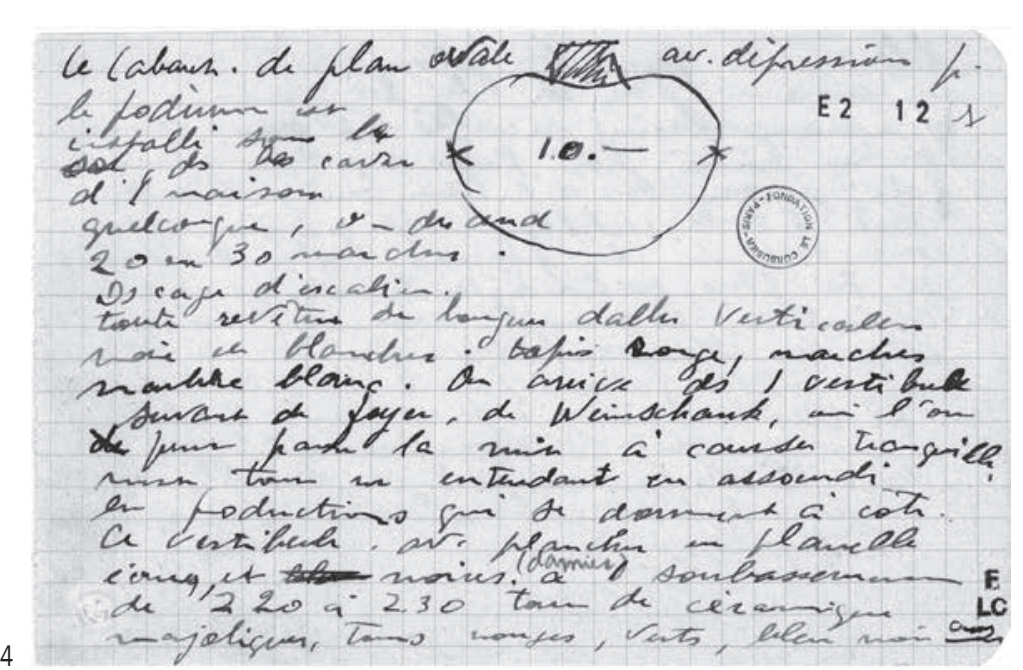

pintor y escenógrafo Fritz Erler'19 para la obra de Shakespeare le decepcionan y critica la sala, dibujada en planta y sección longitudinal (figura 3), aunque alaba su proporcionado exterior ${ }^{20}$. El desencanto le lleva a encontrar incomparablemente mejor el cabaret vienés El Murciélago Fledermaus- (1907) de Josef Hoffmann que ha visitado en el invierno de 1907. Afirma: "En resumen, el Fledermaus / cabaret de Viena era / incomparablemente mejor tanto / del punto de vista de la arqui-/ tectura y de los materiales/ empleados, como de la puesta/ en escena"121. LO describe profusamente junto a un pequeño dibujo en una carta dirigida a su maestro $L^{\prime} E$ Eplattenier ${ }^{22}$. Conviene destacar este hecho porque el cabaret es una sala, instalada en un sótano, de forma parecida a un riñón, con un podio como escenario en el lado curvo más corto (figura 4).

Más laudatorios son los comentarios al Gran Auditorio y, especialmente, a la Sala de las Fiestas -Grosse Aula- de la Universidad (1906-1910) de German Bestelmeyer; esta última le llama la atención por su forma oval, como la denomina, aunque en realidad es un rectangulo

rematado por sendos semicírculos en los lados cortos. Continúa el 8 de octubre con los elogios sobre el Teatro de Marionetas, inspirado en la arquitectura del setecienIos, que Paul Ludwig Troost²3 acaba de construir ese mismo año en el parque Bavaria, un tema al que se enfrenta Jeanneret durante su estancia en Laubach al construir un teatro con este cometido24.

Después, ya en Berlín, a la repugnancia que siente en febrero de 1911 ante Fidelio de Beethoven en la Gran Opera -Opernhaus $2^{25}$, similar a la suscitada por las representaciones de la ópera de París, se contrapone la seducción ante Orfeo y Eurídice de Gluck en junio de 1913 con puesta en escena de Adolphe Appia, en la clausura del curso del Instituto Dalcroze en Hellerau, sólo comparable a la que le ha producido dos años antes el servicio religioso dominical en la catedral de Francfort26

Jeanneret habla conocido al escenograto Adolphe Appia en París ${ }^{2}$ y consolida la relación por medio de las

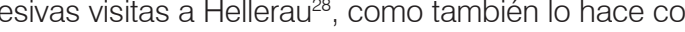

19. El interés por Fritz Erler y la escenogrifafía de Hamlet tiene probabblemente su origen en las conversaciones con William Ritter, autor de algunos artículos sobre este pintor y decorador. Ver De Simone, Rosario: Ch. E. Jeanneret-Le Corbusier Viaggio in Germania 1910-1911. 0p. cit., nota 5, p. 105. 20. Le Corbusier (Ch.-E.J Jeanneret): Les voyageses d'Allemagne. Carnets. Op. citt, carnet 3, 27 de octubre de 1910, transcripción:: entrada [1] 3, p. 83; dibujo: p. 1

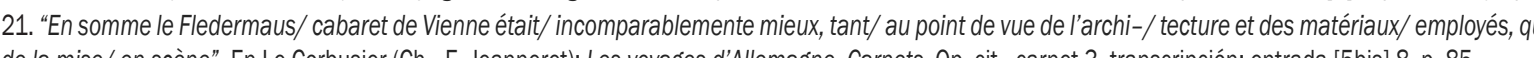

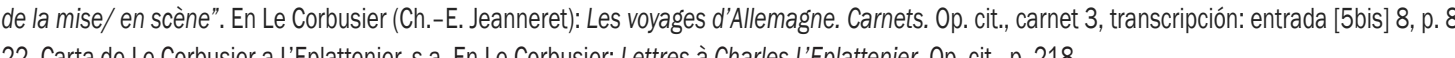
22. Carta de Le Corbusier a LEEplattenier, s.a. En Le Corbusier: Lettres à Charles L'Eplattenier. Op. cit, p. 218

(1934-37. 24. Carta de Le Corbusler a Ritter, final de abrit de 1911, en la que describe su estanciala en Laubach en casa del hermano de su amigo Klipstin. En Grester 25. Le Corbusier (Ch.-E. Jeanneret): Les voyageses d'Allemaggne. Op. cit., carnet 3, transcripción: entrada [81] 86, p. 114; dibujo: pp. $91-92$.

26. Le Corbusier (Ch.-E. Jeanneret): Les voyageses d'Allemagnne. Op. citt, carnet 4, 23 de abril de 1911, transcripción: entradas [48] 50- [52 54, po. 144-146. 27. Carta de Le Corbusier a sus padres, Berlin-Neu-Babelsberg 28 de octubre de 1910. En Le Corbusier: Correspondance. Lettres à sa famille. Tome : 1900-1925. 0p. cit., p. 326

28. Jeanneret aprovecha el 21 de octubre para visitar a su hermano Albert en Dresde, conocer la ciuddad y el distrito de Hellerau, la primera ciudad-jardín
alemana de la que quiere también extraer provecho. Las visitas a Hellerau son en octubre y navidad de 1910 , mayo de 1911 y junio de 1913 . 


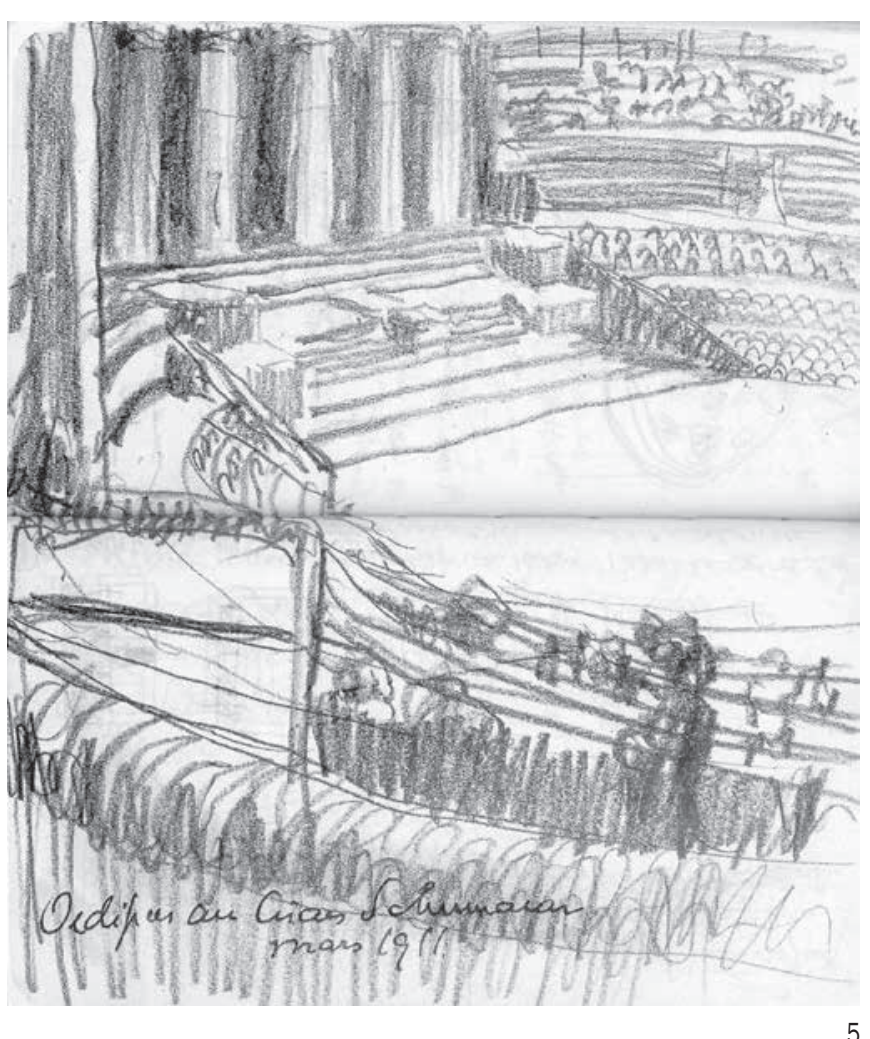

Jacques Dalcroze $e^{29}$. Con la primera visita, que surge durante su segunda estancia en Munich en septiembre de 1910 mientras espera la confirmación escrita para poder trabajar con Peter Behrens en Berlín, y las siguientes tiene la oportunidad de estudiar en profundidad la rítmica corporal y las concepciones escenográficas que Appia da a la masa bajo la luz, plasmadas en sus escritos y diseños operísticos para el teatro del Instituto Dalcroze de la ciudad. Appia las resume en el artículo de junio de 1904 publicado en La Revue de París: "Un objeto no es plástico para nuestros ojos más que por la luz que lo golpea y su plasticidad no puede ser puesta artísticamente en valor más que por un empleo artístico de la luz"30.

Jeanneret, que se impregna de la concepción escenográfica del gran pionero de la escena moderna, que está cautivado por la luz y las culturas antiguas del Mediterráneo en su viaje a Oriente, ensalzando cualquier
5.Le Corbusier(Ch.-E.Jeanneret).Apuntede “Oedipus (6) Edipo Rey con dirección de Max Reinhardten el Cirieo Schumann, Berlín 1910 .

T. Hans Poelzig. Grosses Schauspielhaus, Berlin 1919. Planta.

Hans Poelzig/ Fotoatelier Enst Wasmuth Verlag

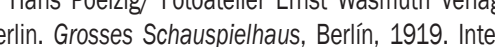
rior de la sala.

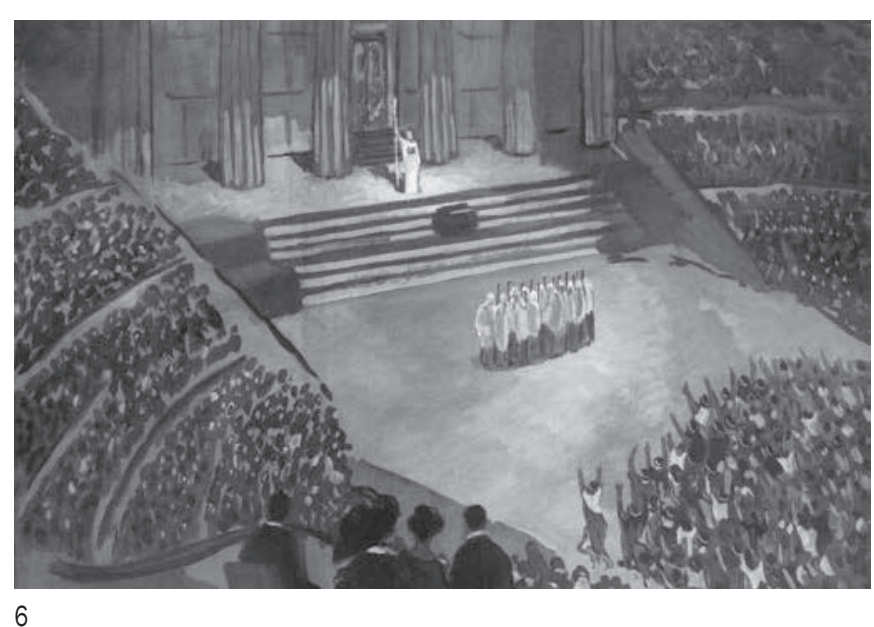

sencilla construcción si está magnificada por el sol, que padece una deficiencia ocular, ese Jeanneret es el que se hace eco de su maestro teatral, sin citarle, cuando quiera expresar la esencia de la arquitectura como "el juego sabio, correcto y magnífico de los volúmenes reunidos bajo la luz"31. Si la semilla de Appia rebrota años más tarde ¿por qué no iba a hacerlo todo lo aprendido en Alemania sobre la escenografía, puesta en escena y teatros cuando tiene que proyectar el Palacio de los Soviets?

En la conversación mantenida en Dresde con Salz mann ${ }^{32}$, Jeanneret le pregunta directamente" $¿ Q u e ́$ es una puesta en escena en la ópera o en el teatro?"33. Obtiene por unica repuesta el lamento por la agonia de la escena contemporánea, a lo cual añade a su interlocutor " $i B r a-$ vo!' Mientras estuve alli, expresé, mi pensamiento desde hace mucho tiempo, nacido y formado, fortalecido con cada nuevo espectáculo al que asisti" ${ }^{\prime 34}$, y apostilla que

29. Su hermano Albert, dedicado a la música, está relacionado con Jacques Dalcroze desde su formación en el conservatorio de Ginebra como alumno y des

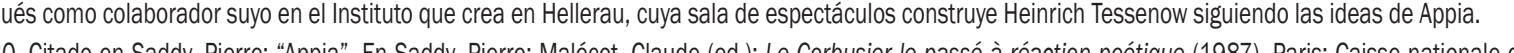
(1990, p.209. 31. Le Corbusier: Hacia una arquitectura (1923). Op. citt, p. 16. La frase fue publicada por primera vez en L'Esprit Nouveau $n^{\circ} 1$, octubre de 1920 32. Alexander Von Salzmann concibe el sistema de iluminación en la sala de espectáculos del Instituto Dalcroze de Hellerau. 33. "Qu'est qu'une mise en/scène à 'opérra ou au/ Theââtre?". En Le Corbusier (Ch. E. Jeanneret): Les voyageses d'Allemaggne. Carnets. Op. cit, carnet 3, trans. cripción: entrada [77] 83, p. 113.

34. "Je lui réspondis: "'rravol!' Car c'était là, exprimée,/ ma pensée depuis longtemps/ née et formée, affermie à chaque nouveau spectacle/ auquel

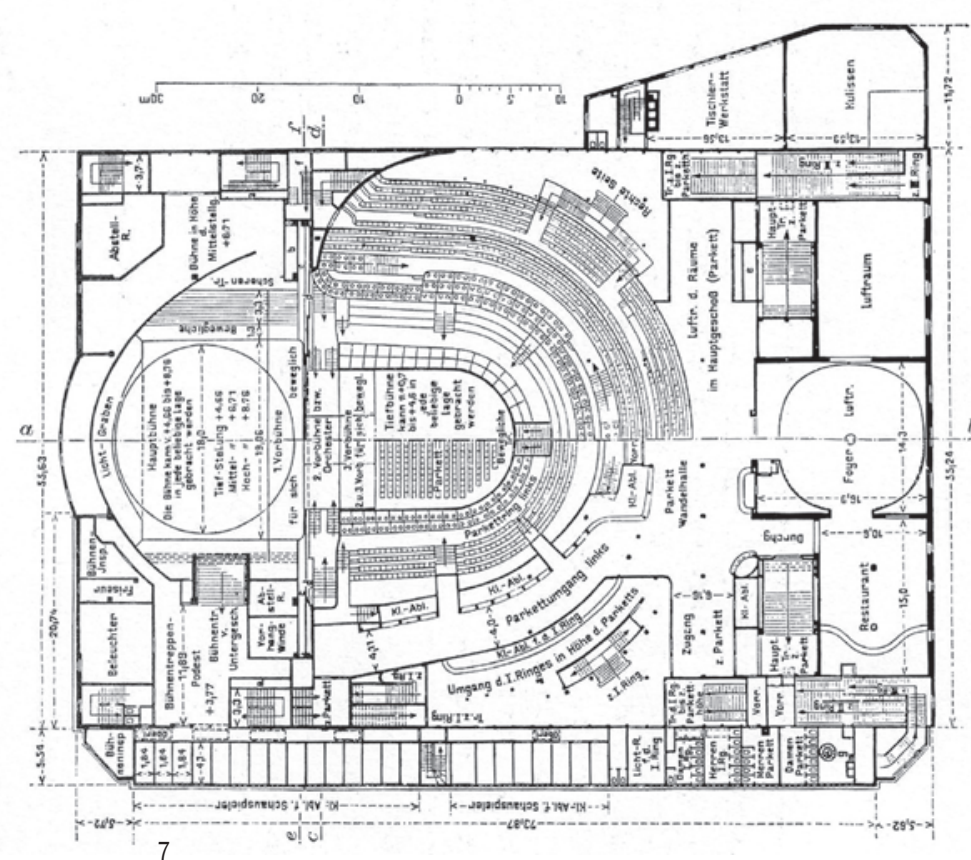

el ritmo y la sala del Instituto $D$

evolución artistica de la época.

Las inmes convicciones que adopta se van gestando con el aprendizaje in situ desde el papel de espectado. En elcanct 3 de su viaje dibuja un apunte que adquiere la dimensiôn de doble página $a^{35}$, una pista o arena rodeada de espectadores con una fachada de columnas como fondo de escena. Al pie del dibujo escribe sucintamente sin ningun otro comentario: "Edipo en e/ Circo [C]/ marzo 1911//36 (figura 5). En la transcripción del carnet de Fondation Le Corbusier se describe la frase como termtnos cripticos, porque no hay aclaraciones en el contexto sobre a que se refiere Jeanneret. Lo que si es evidente es que se ha hecho desde el punto de vista de un especador que espera al inicio de la representación, o en receso de ella debido a la escena vacla de actores. Se llama la atención sobre la falta de referencias a este dibujo en publicaciones nacionales e internacionales, y se insiste sobre la importancia que parece tener para Jeanneret por su tamaño. Para averiguar su contenido, se han colejado las fechas de las representaciones teatrales de ese periodo en Berlín y las fotografías de la época

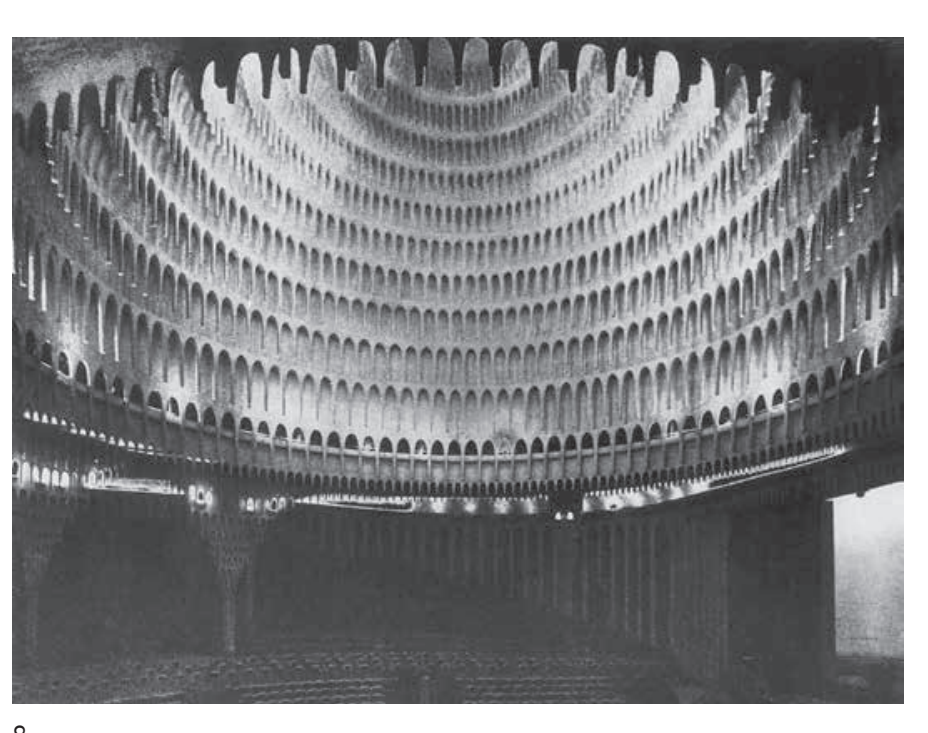

obteniendo de las indagaciones que se trata de la puesta en escena por Max Reinhard de Ealpo Rey de Sófocles con escenografia del arquitecto Alfred Roller, del que Jean neret canta las excelencias de su trabajo en Viena ${ }^{37}$. En representación de la obra que dibuja Emil Oriliks, el coro ocupa el centro visual del espacio, rematado en un extremo por una arquitectura que evoca el palacio de Tebas. elevado sobre una escalinata para proporcionar distintos niveles y profundidades de juego escénico (figura 6). da por Georg Fuchs, de abrir el Volksfestspiele de Munich en sepliembre de 1910, poniendo en escena Edipo Rey en el Palacio de Festivales de Theresienhohe. Queria plasmar su idea sobre un nuevo teatro del pueblo, "Teatro de los Cinco mil" según su denominación, pero la sala del festival carecta del tipo de escenario necesario para la representación de este teatro para las masas. por lo que tuvo que ser remozada para incluir una are na. Cuando la producción se traslada posteriormente Berlin, antes de iniciar una gira europea de gran éxito, se epresenta en un verdadero circo, el Circo Schumann (figuras 7 y 8) -nombre que se encuentra escrito en el

35. En los cuatro carnets de viajes por Alemanii existen un total de tres dibujios a doble página completos: el del carnet 1 es desconocido y los dos del carnet 3

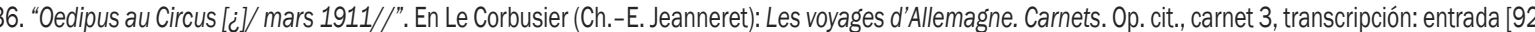
97, p. 119; dibujo: pp. 91-92. También menciona la obra con “demain OEdipus" en una carta a sus padres, 9 de marzzo de 1911. En Le Corbusier: Correspordance. Lettres à sa famille. Tome l: 1900-1925. 0p. citt, p. 352 .

37. "La mise en scène de/ Vienne, sous la direction de/ Alfred Roller, peut encore enchanter/ par ' 'art très grannd et très/ pur s'y manifeste". En Le Corbusier

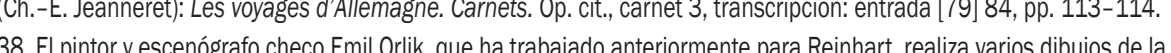

.

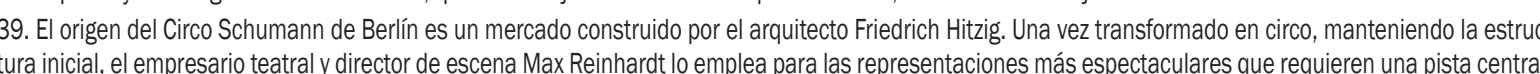
hasta que le encarga su renovación a Hans Poelizigy se convierte en el Grosses Schauspielhaus, que abre sus puertas en 1919 hasta su transformación por los nazis en 1933, después alberga differentes usos y finalmente es demolido en 1988. El i imponente exterior rojo y los fantásticos foyers vegetalales preceden a la cúpula de estalactitas, colgada sobre una escena que se debate entre el espacio central del anfiteatro y el escenario panorámico direccional 


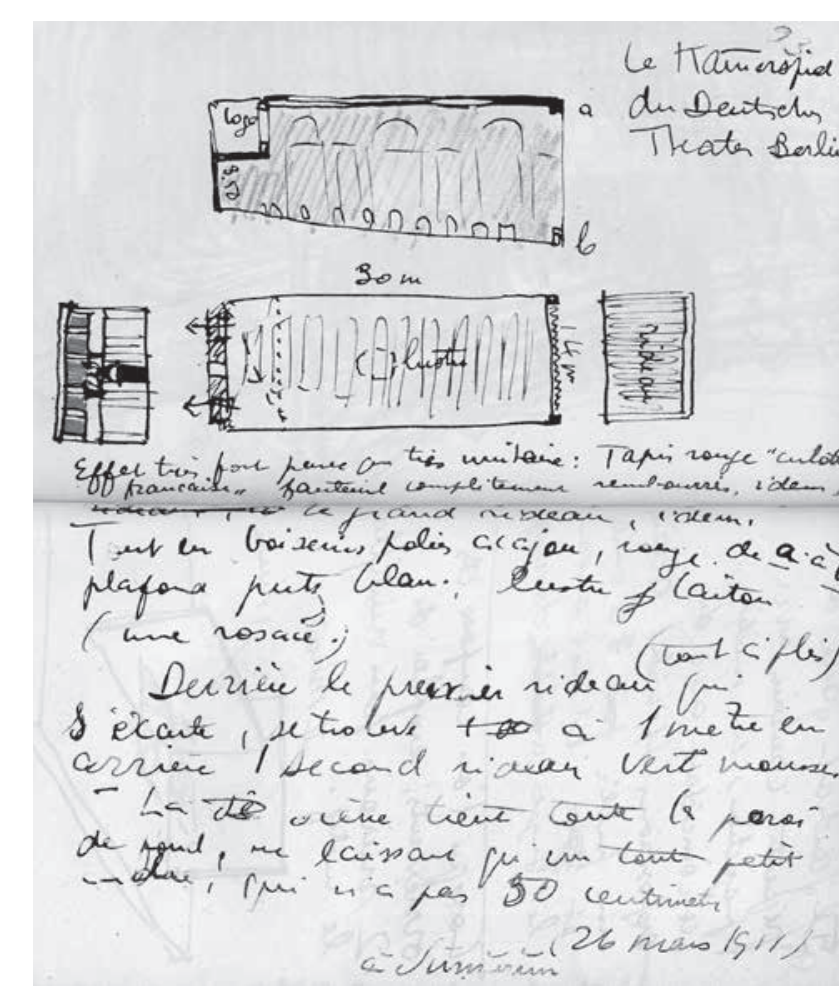

9

carnet y que debe ocupar el lugar de la interrogación en la transcripción-

De los dos espectáculos teatrales que aborda Reinhardt, la representación de cámara ${ }^{40}$ y la de masas, es unicamente en la segunda donde considera "espectáculo" el ofrecido tanto por los actores como por el públicoti Por esa razón, el modelo al que acude para organizarlo es el circo, a falta de edificios preparados para ello, que con su pista o arena permite ver y ser visto, saca del confinamiento la escenificación de la obra y acerca el actor al público. La capacidad de dirigir grandes masas en un amplio espacio y el factor de cohesión entre público e intérprete manifiestan la fuerza de su dirección.

No es baladi la referencia a la obra de Reinhardt al tipo de espacio que busca para sus espectaculares representaciones, por cuanto Jeanneret registra la experiencia del juego escénico con este circo, que tiene una doble significación: el manejo dramático de las grandes masas ${ }^{42}$ y el alejamiento de los teatros hacia otros lugares donde no exista separación de sala y escena, una constante y reivindicada aspiración de los renovadores del teatro desde finales del siglo XIX.

La Sala de Cámara -Kammerspiel- del Deutsches Theater de Berlín de William Müller tiene su lugar en la siguientes dos páginas del carnet 3 de Le Corbusier, a continuación del dibujo sobre Edipo Rey. Dibuja la planta una sección longitudinal y dos transversales de la sala (figura 9) y parece describir una escena de otra obra dirigida por Reinhardt, menciona "I (26 marzo 1911/ en Sumurun//"43. Todo un variado repertorio de representaciones y de arquitecturas dedicadas al espectáculo orientan la actitud de Jeanneret hacia el rechazo de las formas de la representación naturalista. En el informe Étude sur enoureme, que redacta a inal de su paso por Alemania por encargo de la Escuea de Arte de La Chaux-de-Fonds y siguiendo las notas ya comentadas de su carnet, de nuevo alaba la exquisita sala del Teatro de Marionetas de Troost, el modelo-tipo de teatro del Künstler Theater $y$, expresamente, vuelve mencionar a Reinhardt"4 ${ }^{4}$. Asi pues, el aprecio por su obra queda patente, y el circo, espectáculo que obtiene las simpatias del publico y el interés de la vanguardia artística europea, llega a ser un tema obligado que se introduce desde el primer número de la revista L'Esprit Nouveau con el artículo "Le Cirque, art nouveau" de la escritora dadaista Céline Arnauld ${ }^{45}$ 40. Su apuesta por la "representación de cámara", al contrario que la de masas, se desarrolla en espacios y con público reducidos, requiriendo un entrena-

41. La constante referencia que hace Reinhardt al teatro antiguo parte de una contradicción: se toma como modelo el anfiteatro, aun cuando en él no se desa rrollan dramas sino que es un lugar de juegos. En Posener, Julius ( $\mathrm{A}$ cargo de): Hans Poelzig. Scritti e opere (1970). Milano: Franco Angeli, 1978, p. 169. 42. La teatralización de la comunicación, con el uso dramático de las escenas de multitudes, fue imitada por todas las ideologías pollticas de la primera mitad del siglo XX. La herencia de Reinhardt, de un escenario inmenso para el teatro del pueblo, se conviritió en sus manos en una convención del teatro de masas. En una época caracterizada por la movilización politica popular, el teatro para grandes concentraciones humanas no puede verse como patrimonio exclusivo de ninguna de estas ideologias.

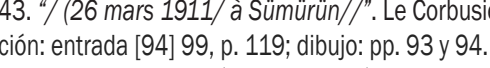

44. Le Corbusier (Ch-E.. Jeannerett): Étude sur le mouvement d'art décoratiffen Allemagnne. La Chaux-de-Fonds: FLC, 1912, pp. 31, 32 y 33.

45. Amauld, Céline: "Le Cirque, art nouveau". En 'EEsprit Nouveau. No 1, 1920, pp. 97-98. El contenido de la revista era de artes, Itras y ciencias, con secciones de teatro, espectácullos y deportes.
Años más tarde, en 1948, ya convertido en Le Cor- la ciudad ${ }^{47}$. Como todos los montajes de Reinhardt estos busier, pronuncia en la Sorbona su más famosa y espećífica conferencia sobre el tema, titulada "El teatro espontáneo" $" 46$. La forma de exponer sus experiencias con el teatro, en principio, parece contradictoria. Al contrario de lo esperable en alguien dedicado a la arquitectura, y través de una hábil estructura circular de la narración, empieza y termina con la defensa del teatro espontáneo, $n$ sin antes recorrer todo tipo de espectáculos. Comienza con el origen de la idea, durante su segundo viaje a Bras en 1936, ante la pregunta de Gustavo Capanema, ministro brasileño de Educación y Salud pública, sobre cómo concebir un teatro moderno en Rio. Le Corbusier observa a variedad y mezcla de razas que convierten la calle en un verdadero teatro, lo que le da pie a la defensa de u eatro esencial de expresión y medios elementales, con maquinaria escenográfica reducida a simples tablados, los mismos que se encarga de levantar en las cubiertasjardín de las Unités para disfrute de los habitantes con sus propios espectáculos.

Desde el punto de partida, de la defensa del teatro despojado de cualquier artificio, se va incorporando paulatinamente la arquitectura, con el teatro sencillo que se produce en los clubs de los centros agrarios del $V$ lage cooperatif, en donde empieza a intervenir la escala humana de la sala. Luego pasa a hablar de la regeneración teatral al sacarlo a la naturaleza, se acuerda de Reinhardt y dos obras de Shakespeare dirigidas por é a las que asiste tiempo atras: El sueño de una noche de Campo San Trovaso de Venecia, una pequeña plaza de dos rompen también con la escena tradicional, más aún cuando dichas obras se representan en exteriores. A los do de naturaleza en Berlín, contrapone el entusiasmo por a ubicación del público en medio de la plaza veneciana mientras la representación se desenvuelve a su alrededor Con la arquitectura como fondo y desde ella, los actores interpretan y el público se siente partícipe de la obra. Se pregunta Le Corbusier cual es la causa de que estuviera tan bien y reconoce que Existen lugares de consonancia matemática que llamo lugares de acústica visual"'to que no van a consistir en determinar en valor absoluto et tamano de la sala, ni en decantarse por un espectaculo concreto, sino en llegar a un mutuo acuerdo, según aclara en la discusion posterior: "No hay una dimensión fija. Creo que hay relaciones precisas"

Ante la necesidad de proporciona sala y tipo de representación trae a colación el Palacio de los Soviets y recuerda su protesta por una sala de tal magnitud, tan grande como la Plaza de la Concordia de París, dice, en la que seria imposible ver nada que se representase. Para abordar este inconveniente, $y$ haciendo de la necesidad virtud, explica: "He diseñado una pequeña teoría defendible, pero la experiencia no ha podido confirmarla porque el Palacio no fue construido. He aqui la teoria: si todo está de acuerdo, se creara una consonancia o -repito- una acustica visual similar a la de los sonidos" ${ }^{\prime \prime 0}$. Básicamene consiste en que para compensar la pérdida de visión tir la presencia de auditorio, de nuevo acude a Reinhardt, que exista un

46. Le Corbusier. "Le théätre spontane", conferencia pronunciada el 11 de diciembre de 1948 en la Sorbona con ocasion de la primera sesion del Centre $d^{\prime \prime E t u d e s ~ P h i l o s o p h i q u e s ~ e t ~ T e c h n i q u e s ~ d e ~ T h e ̂ a ̂ t r e . ~ E n ~ V i l i l i e r s, ~ A n d r e ́ ~(P r e s . ~ p o r): ~ A r c h i t e c t u r e ~ e t ~ d r a m a t u r g i e i . ~ P a r i s: ~ F l a m m a r i o n, ~ 1950, ~ p p . ~ 149-168 . ~}$

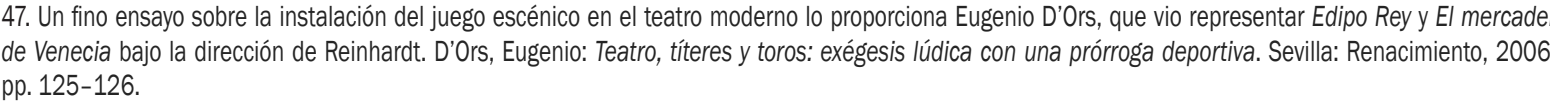
pp. $125-126$.

48. Il existe des lieux mathématiques de consonante que j'appellerai des lieux d'acoustique visuelle". Le Corbusier: "Le thếtre spontané". En Vililiers, André

49. In'y a pas de dimension fixe. Je pense qu'li y a des rapports justes". Intervención de Le Corbusier en el coloquio de su conferencia. En Villiers, André (Pres,
por: : Architecture et dramaturgie. Op. cit., p. 161. 50. "Je me suis dessiné une pettie théorie défendable, mais l'expérience n'a pu la confirmer parce que le Palais n'a pas été construit. Voici la théorie: si tou est mis en accord, on créera une consonance ou -j'y reviens- une acoustique visuelle semblable à celle des sons". Le Corbusier: "Le thêâtre spontané". En 
concentración plástica que produzca solidaridad visual, la que se encuentra en el teatro circular: "Es la fuerza de los circos, las arenas, las plazas de toros. Alli, en esta solidaridad visual está lo bien hecho de las reuniones. Si alli [el público] no se ve, esto hace el hitterismo"5.

Quizá la parte más controvertida sea la que sigue donde tiene más dudas, en la que deja sin respuesta más preguntas, alude a los espectáculos a gran escala, a escala urbana, como los posibles para las salas colosales:

"De todos modos, por supuesto este tipo de salas no pueden senir mas que para ciertos espectáculos. Se ha inventado para ellas los desfiles de tanques y dirigibles, de aviones, bajo pórticos lo suficientemente grandes. Eso Campos El'seos, eso divierte astiles del 14 de julio en los Campos Elseos, eso divierte a la gente.... Esta es una cara ción. Yo no decido. cion. Yo no decido. No se tiene el derecho a decidil. Todo es posible, si está bien hecho, si tiene exito. Quizáalguien vengativas encontrando el espectáculo adecuado"5?

Casi a renglón seguido se refiere a un gran centro de fiestas populares para 100.000 espectadores, en el que stuvo trabajando, y enumera los posibles espectáculos: "Para mover a tanta gente, se necesita un fin. Propuse un uso múltiple: tribuna politica, porque, en definitiva, es el gran 'papá' de los tiempos modernos; alli los oradores podrán oratoriar, a continuación, propuse que haya teatro, despues destiles para complacer a todo el mundo: al final, fiestas gimnásticas gigantes ¿Por qué no? Se llega a uno de los límites extremos donde entra en juego

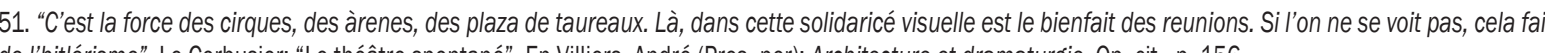
de l'hitlérisme". Le Corbusier: "Le theâtre spontané". En Vililers, André (Pres. por): Architecture et dramaturgie. Op. citt, p. 156.

52. "Quoi qu'il en soit, ces sortes de salles ne peuvent se prêter qu'à certains spectacles bien entendu. On a inventé pour elles les défilés de tanks et de diritgeables, d'avions, sous des portiques assez grands. Ça amuse beaucoup de gens. Les défilés du 14 juillet sur les Champs-Elysées également, ca amuse les gens... C'est une face de la question theatrale qui laisse planer une interrogation. Je ne tranche pas. On n'a pas le droit de trancher. Tout est possible, sic'es bien faitit, sic'est réussi. Quelqu'un peut-être dissipera cette inquietude quej Jai des distances vengeresses en trouvant le spectacle idoine". Le Corbusier. "Le

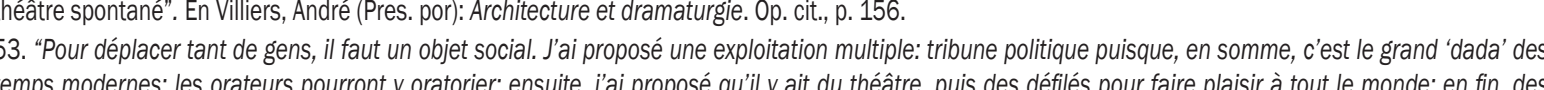
Etes symniques gigantesques. Pourauoi pas? Vous atteigrnez une des limites extrêmes où entre en jeu le fluide des foules. Evidemment c' c'est là une puissance

convidérable". Le Corbusier: "Le theâtre spontané". En Vililers, André (Pres. por): Architecture et dramaturgie. 0p. cit,, pp. 156-157.

54. Intervención de Le Corbusier en el coloquio de Barsacc. En Vililers, André (Pres. por): Architecture et dramaturgrie. Op. citit, p. 18

55. Intervención de Le Corbusier en el coloquio de Barsacc. En Villiers, André (Pres. por): Architecture et dramaturgie. op. cit, p. 182

56. En el proyecto de Museo del Siglo XX en Erlenbach (1963) diseña un teatro, escena y auditorio, al aire libre. alguno de los ponentes, pero también saca a colación las dos caras de la historia del espectáculo: el constreñimiento y la apertura al exterior, cuyo origen en su obra se remontaal Palacio de los Soviets. A su vez, dentro del constreñimiento, el circo y la caja de milagros ${ }^{57}$, amalgama y pregnancia del volumen, centro y límite, conviven en su discurso confeccionando un dualismo $0^{58}$ que no puede eludir. La ortodoxia argumental de numerosos ensayos, que fijan la ligazón de su arquitectura con el teatro y cine exclusivamente en la formulación de la bôte à miracles, suele olvidar este otro discurso subliminal y alternativo del circo que también va tejiendo sobre la misma urdimbre del espectáculo. Apenas tres años separan la definición de la boite a miracles vertida en el encuentro teatral de su primer dibujo ${ }^{59}$, acompañante mudo de los proyectos para Ahmedabad, Tokio o Chandigarh. Bajo la forma prístina y desnuda de un prisma rectangular, la minuscula puerta recibe a una legion de diminutos espectadores que acuden a la caja de sueños, una cajón de sastre en el que casi todo cabe, todo menos el cine: "conferencias audiovisuales, música, danza, teatro y juegos electrónicos" encuentro teatral rechaza la sala de doble fin, valida para teatro $y$ cine ${ }^{-1}$ y parece que el cine no se plantea como posibilidad, de hecho, al final de su ponencia, admite

57. El circo y la caja de milagros de Le Corbusier tienen similitud con las dos formas del espacio teatral de Ettienne Souriau, que en el mismo encuentro teatra

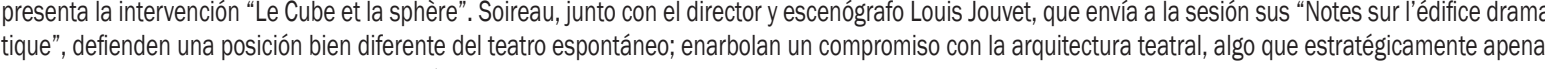

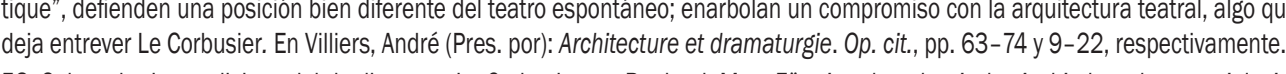
58. Sobre el origen religioso del dualismo en Le Corbusier ver Raphael, Max: Für eine demokratische Architektur: kunstsoziologische Schriften. Frankfurt: S. Fischer, 1976, p. 47.

59. Publicado por primera vez con la conferencia "El corazón como punto de reunión de las artes", pronunciada en 1951 en el marco del CIAM VIII celebrado Lund Humphries, 1952, p. 52.

60. El dibujo de la boîte à miracles está publicado por segunda vez como "prolongements" del Museo de crecimiento ilimitado en la lámina 11 del Museo de Siglo XX, donde se enumeran las actividades contenidas. En Le Corbusier et son atelier rue de Sèreses 35. OEuvre complète 1957-1965 (1965). Zurich: Les Éditions d'Architecture, 1995, p. 170

61. Intervención de Le Corbusier en el coloquio de su conferencia. En Vililiers, André (Pres. por): Architecture et dramaturgie. Op. cit., p. 167. 62. "J'aime beaucoup le cinéma. Je n'y vais presque jamais", Le Corbusier: "Le theâtre spontané". En Villiers, André (Pres. por): Architecture et dramaturgié.

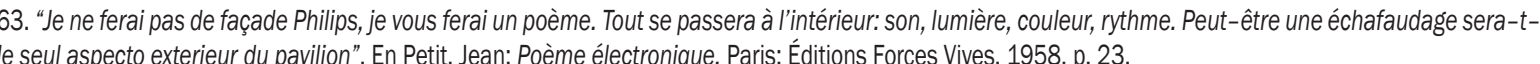

64. La interpretacicín que se hace aquí no es excluyente de la que Fernando Quesada plantea cuando, basándose en el dibujo 32059 de la FLC, propone la

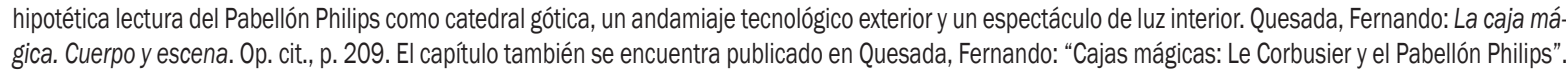
En Massilia: anuario de estuduios lecorbusierianos, 2002, p. 184.

con desvergüenza: "Me gusta mucho el cine. No voy casi versal de Babellon Philips (1958) para la Exposición Uni-

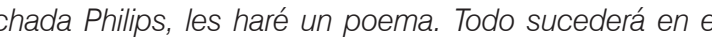
ela construida?64 Un circo en toda regla, que acoge a co政

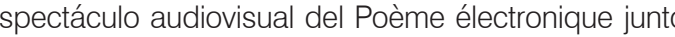
izada. De este modo, la carpa del circo y in e 

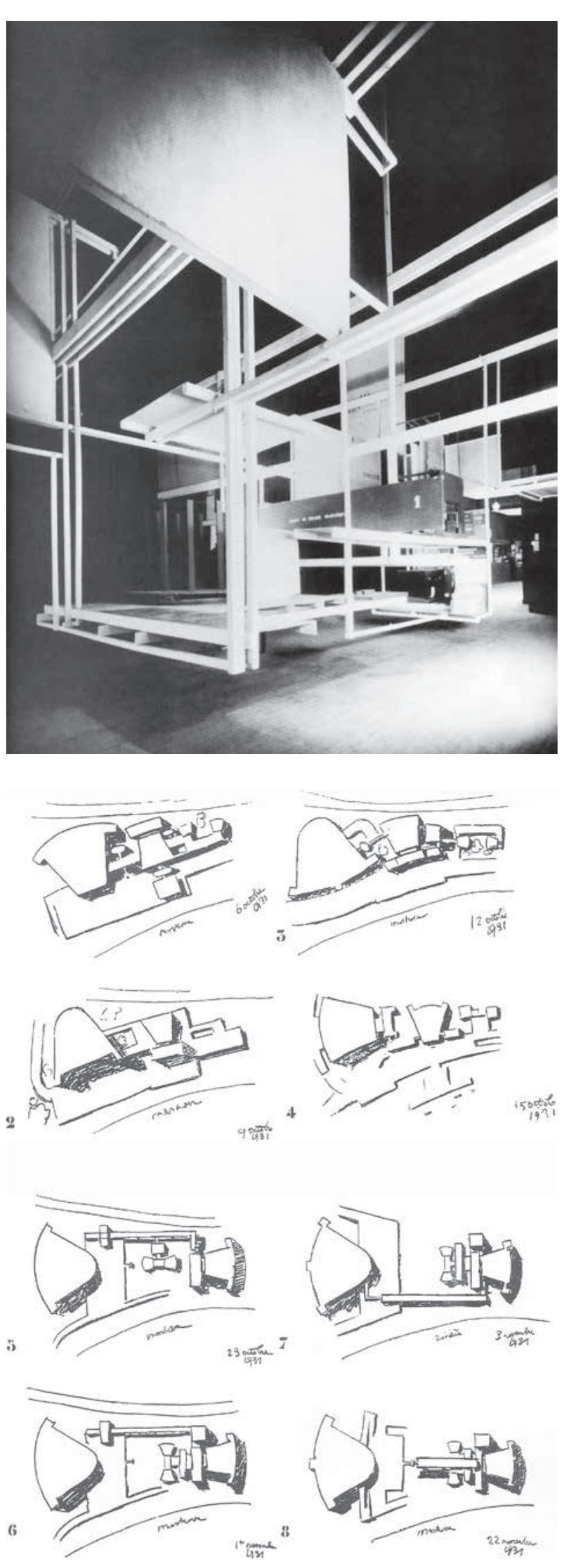

13. Frederick Kiesler. Ciudad en el espacici sall "El arte del teatro en Austria", Exposis
tion des Arts decoratifs et industriels dernes, Grand Palais, París, 1925

14. Le Corbusier y Pierre Jeanneret. Project to del Palacio de los Soviets. Evolución, 19 de diciembre de 1931. el espacio-Raumstadt- en la primera planta del edificioio ${ }^{72}$ (figura 13), nivel donde se hallan las salas soviéticas. Sorprendentemente el contenido no corresponde a las salas de arquitectura de su país, montadas por Oswald Haerdtt, sino a la sala "El arte del teatro en Austria" para la que recibe el encargo, que se sitúa colindante. La pregunta capciosa que lanza Le Corbusier a Kiesler ${ }^{13}$ sobre la ciudad sólo puede demostrar la pugna por la primacía de quien quiere ser el paladín de lo moderno a los ojos del mundo. La fria documentación dibujada de los dioramas de la Ville contemporaine de 3 millions d'habitants (1922) y el Plan Voisin (1925) en el interior del Pabellón de L'Esprit Nouveau contrastan con el efectismo de Ciudad en el espacio. La distante información de aquellos son todo lo contrario a la inmersión en el montaje de ésta, donde la puesta en escena se experimenta directamente por el visitante para transmititile una sensacion

Tan aclamada llega a ser la sala de Kiesler como las usas entre la critica especializada, pero ninguna deja rastro en el libro L'Art decoratif d'aujourd hui (1925) que Le Corbusier publica sobre la exposición, poco más que la liustración del decorado pictorico de Picasso para la obra Tricorne $(1919)^{74}$ de los Ballets de Diaguilev tiene relación con la escena. LFalta de interés? no parece, más bien que su naturaleza le impide alabar públicamente lo interesante entre sus coetaneos, queda para la historia la aclaración De igual modo en la exposición internacional están incluidos los mejores y más novedosos teatros; además la construcción del Teatro de la exposicion es confiada a Auguste y Gustave Perret, arquitectos con experiencia en el tema después haber erigido el Teatro de ChampsÉlysées (1913), del cual Le Corbusier se deshace en elogios tiempo atrás: "El teatro de los Campos Eliseos es su obra. Y para mí, es la más grande arquitectura desde hace tiempo escena tripartita, basada en la del teatro de Henry Van de Velde para la exposición de la Deutscher Werkbund (1914) de Colonia, de cuyo destronamiento por los Perre se había alegrado Le Corbusier', sin por ello dejar de visitar el edificici" ${ }^{71}$. Con la convergencia de tantas obras en la exposición, de tanto interés juvenil por la arquitectura del teatro, se reitera la extraneza de su total ausencia en el libro citado. Realmente es un libro de ataque, en pocas palabras, todo lo que introduce es para denigrarlo.
Siguiendo la línea de lo que no dice Le Corbusier, y propuestas previas y a eje al final como remate del testero para introducir el siguiente tema, se acude al dibujo del 19 de diciembre de 1931 con las etapas por las que atraviesa el desarrollo del proyecto del Palacio de los Soviets, entre el 6 de octubre y el 22 de noviembre del mismo año, redibujadas para servir de aval a la solución fina adoptada (figura 14). En las cuatro primeras soluciones, el conjunto es una amalgama de volúmenes agregados que varian fundamentalmente por la orientación de las dos salas principales, pero que tienen en común disponer de un foro-plataforma en primera línea de la ribera. En as cuatro soluciones siguientes, las dos grandes salas se desplazan centrifugamente a los extremos y se vacía el centro, variando su delimitación, al fondo o al frente. con un bajo bloque de oficinas sobre pilotis, el cual se reubicará definitivamente en el eje de simetría sin llegar recorrerlo totalmente.

El foro-plataforma paralelo al rio del primer grupo, se disocia y separa de las salas en el segundo. La plataorma adquiere estatuto propio, dejando de ser la base de todos los edificios para pasar a adherirse sólo a la gran sala. Por consiguiente, el foro ha terminado casi por diluirse, al ocuparse parcialmente el eje con la fina barra administrativa sobre pilotis, y la plataforma se ha convertido en una platea elevada y flotante para el público del gran auditorio al aire librers, que tiene a la tribuna de oradores como escenario, descentrada en alguna de las

de la barra administrativa

Sin embargo, a la vez, la plataforma es escenario en sí misma al poder acoger las "manifestaciones teatralizadas" habituales en los primeros años revolucionariost La masa se convierte en actor y discurre por las rampas que conectan el suelo de la ciudad con la plataforma aún más allá, por aquellas otras que lo hacen hacia e escenarno del interior de la gran sala, para despues sair por las opuestas, permitiendo una gran diversificación del lugar de la representación, como ocurre en las plataformas y entresijos del El cornudo magnifico. También la masa puede desfilar por el toso" entre la plataforma y la tribuna de oradores, mientras el público general asiste, dividido en dos bloques jalonando al orador, a los acontecimientos que se desarrollan en el exterior. Clertamente, la suma de los aforos de la gran sala interior y los niveles exteriores en un evento hubiera acogido a mucho más que multitudinarias concentraciones humanas. El proyecto para el Palacio de los Soviets se erige asi en ese lugar de acustica visual apropiado a la escala y flujo de los grandes especláculos de y para las masas, como parece deducirse de sus palabras en el encuentro teatral años más tarde, que discurren por y a través de una gigantesca máquina de actuar

El tandem constituido por la tribuna de oradores y la platea-escenario de la plataforma en la sala exterior se
72. Kiesler consigue cambiar la ubicación de planta baja a primera. En Reichlin, Bruno: "The City in Space". En AA.W.: Frederick Kiesler, Artiste-architectearis. Centre Georges Pompilou, 1996, p. 1 .

政

74. Nombre francés del ballet El Isombrero de detres picos de Pedro Antonio de Alarcón con músicica de Manuel de Falla. En Le Corbusier: LArt decoratifif'aujourd'hu Perret, un diseñarion, 1996, p. 158. Además de la escenografíá, en el libro se encuentran ilustraciones del vestibulo del teatro de Champs-Elysees de Ay .

T5. Canta a linam Ritter, 9 de junio de 1917. En Le Corbusier: Choix de lettres. 0p. citt, p. 115.

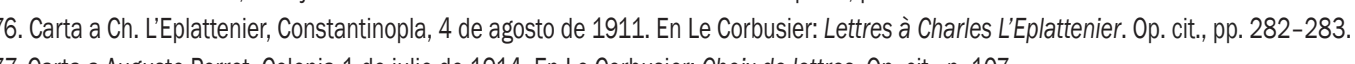

77. Carta a Auguste Perret, Colonia 1 de julio de 1914. En Le Corbusier: Choix de lettres. Op. citt, p. 107.

78. Cohen lo sugiere escuetamente. Cohen, Jean-Louis: Le Corbusier et la mystique de 'URRSS. Op. cit., p. 214

79. Entre los espectaculos de masas que se organzaron, es decir, donde estas eran parte activa, dirigidos también a las masas, se encuentran las represenr por el pintor y escenóórrafo Yuri Annenkov, que narra los acontecimientos históricos que se produjeron en Petrogradodo (San Petersburgol) durante la revolucución de octubre de 1917. Fue protagonizzada por la propia población en los Iugarese en que sucedieron los hechos, mientras los espectadores asistieron a la repre sentación ubicados en dos bloques simétricos al eje de la plaza del palacio. Sin ser exclusivos de las representaciones teatrales o los desfiles, era frecuente intercalar mítines de importantes personalidades dirigidos a estas multitudes. 

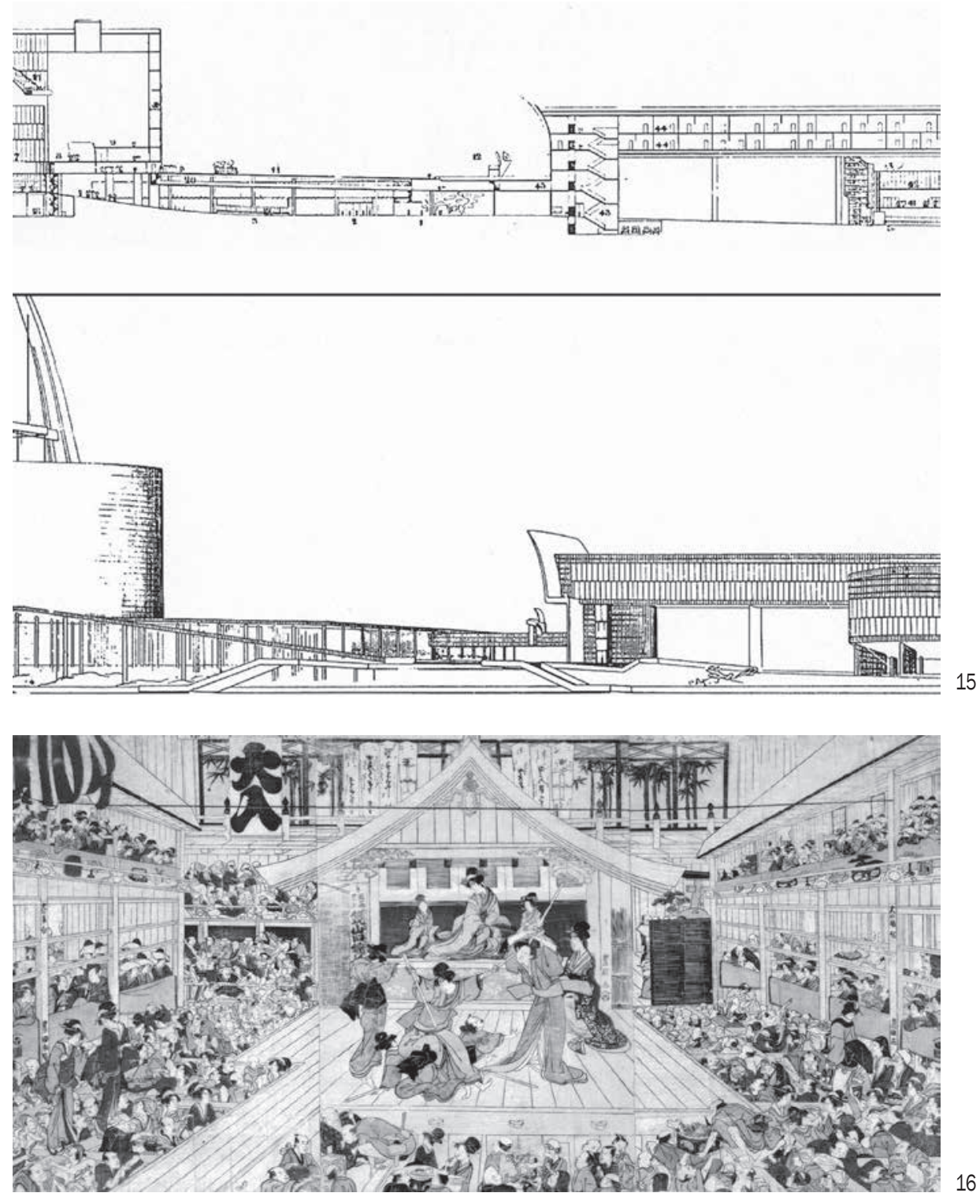

15. Le Corbusier y Pierre Jeanneret. Proye les del enlace en las secciéón y alzado. 16. Utagawa Toyokuni I. Grabado del Tea de Kabuki Kawarazaki-za con el "camino fllorido" o hanamichi, periodo Edo 1800 17. Le Corbusier, Sergei Eisenstein, Andre 18. Eisenstein y el actor japonés Ichika
Sadanii II en Moscú, agosto de 1928 . convierte en el nudo gordiano de lua todo se expande. Pero lo que llama poderosamente la atención es e escueto y fińsimo cordón umbilical que las enlaza junto con sus respectivas salas interiores, en el eje de la composición (figura 15). Un eslabón ridículo que casi parece desgarrarse ante la imponente fuerza expansiva de las masas radiales centrífugas. Consiste en un finger que une la tribuna del orador a la plataforma, con recorrido interior y superior por su cubierta convertida en una pasarela de comunicación entre ambas. Este elemento desempeña el mismo pape en la escenografía del Palacio de los Soviets que el denominado hanamichi o "camino florido" del teatro japonés del Kabuki.
El descubrimiento de teatro de Japón en Occidente el contexto de apreciación de todo aqueo relacionado con el arte japonés, corriente denomina(jomonismo'so. Una de las vías de conocimiento e sus artes son las giras de compañías teatrales que reorrieron Europa, gracias a las cuales se difunde uno de los géneros dramáticos del teatro japonés, el teatro popular del Kabukisi Sus representaciones se desenvuelven sobre el escenario y sobre una parte extra una plataforma larga que se instala a su izquierda y discurre través de la audiencia, uniendo el escenario con la parte posterior del teatro, es el denominado hanamichi o "camino florido" (figura 16).

Aunque el hanamichi no se usa en las giras europeas del Kabuki tal y como consta en las fotos de la época sin
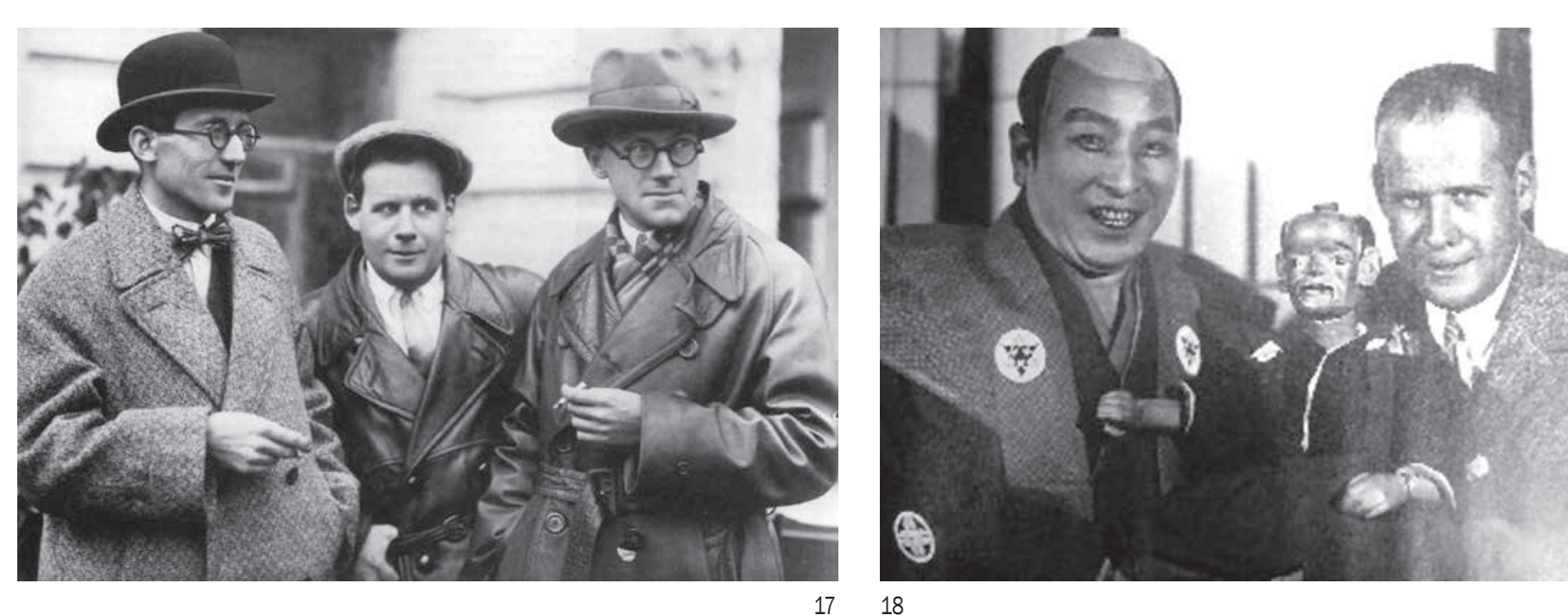

embargo, se da a conocer a través de las publicaciones de finales del XIX sobre el teatro japonés, lo que contribuye a que los europeos encuentren en este elemento un puente entre actor y espectador y se sirvan de él para establecer una nueva unidad entre ellos. Una idea recu rrente en Meyerhold y Reinhardt, dos grandes nombres de la dirección de escena soviética y alemana, que mue ve al austriaco a probarlo por primera vez en 1910 con la obra Sumurún de Friedrich Freksa para la Kammerspie de Berlín en 1910, la misma que cita Le Corbusier en su comentarios sobre esta sala del Deutsches Theatere. Cuando Le Corbusier se reúne con el cineasta senstein, durante su primer viaje a Moscú en octubre de 1928 (figura 17), acaba de actuar en agosto la compañía de Kabuki de Ichikawa Sadanij II de gira por Rusia, actor aponés al que van a conocer Stanislavsky, Meyerhold y Eisenstein (figura 18). Aun ya habiendo elegido dedicarse definitivamente al cine, Eisenstein sigue interesándose por su primera actividad teatral, en la que ha formulado

su teoría del "montaje de atracciones", y por todo lo re el teatro occidental que pueda compararse al Kabuki espectáculo más parecido lo encuentra en el deporte: "Los japoneses nos han enseñado otra forma de coniunto extremadamente interesante -el coniunto monístico- en el cual el sonido, el movimiento, el espacio y la voz no se acompañan (ni siquiera paralelamente) el uno al otro, sino que funcionan como elementos de igual importancia. La primera asociación que nos viene al pensamiento observando el Kabuki es el soccer, el deporte más colectivo y de conjunto

Grandes admiradores del teatro Noh y Kabuki, y Eisenstein iniciado en el idioma, aprovechan cuanto encuentran en el teatro nipón para suplir las carencias que detectan en el ámbito europeo. El nivel de apropiación es tan importante que Eisenstein llega a exclamar: "iMeJeld ha saqueado todo lo que puede ser útil del teatro

80. El teatro oriental, especialmente el de Japón, ejerció gran influencia en las vanguarcias artísticas de finales del siglo XXX y primeras décadas del XX. Sin e

81. El teatro popular japonés o Kabukil llegó en 1899 a diversas ciudades de Estados Unidos de la mano de la compañía de Kawakami Otojirô, actor y marido Francia, cosechando gran éxito en la Exposición Univiversal de París de 1900, así como Gran Bretaña (Londres). España (Madrid, Barcelona) Austria VViena) Rusia (San Petersburgo). Italia (Génova). Alemania o Polonia.

82. Le Corbusier (Ch.-E. Jeanneret): Les voyageses d'Alllemaǵne. Carnets. Op. cit., carnet 3, transcripción: entrada [94] 99, p. 119.

83. Su interés por los procedimientos de expresion visual se inicia cuando entra en contacto con el teatro al ser nombrado en 1921 asistente de decoración

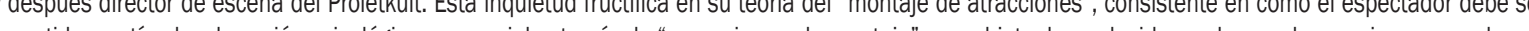
sometido a estimulos de acción psicológica y sensorial, a través de "mecanismos de montaje", con objetto de producirle un choouue de emociones, para lo que primer artículo publicado, aparecido en la revista Lef $\left.n^{\circ} 3,1923\right)$. En El Sentido del cine. Madrid: Siglo XXI, 1999, pp. 172-174. En la misma dirección y apoyán dose en el ideoorrama, elemento básico de la escritura japonesa, establece el concepto medular de su posterior teoría del montaje cinematográfico. Elisensten Sergei: "El principio cinematoografico y el ideograma" (1929). En Teoría y técnica cinematogŕáticas. Madrid: Rialp, 1999, pp. 83-100.

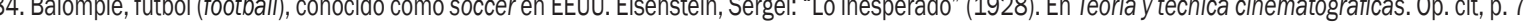

85. Eisenstein, Sergoi: "Lo inesperado" (1928). En Teoría y técnica cinematográficas. Op. cit., p. 74. 
y la poesía de Japón la base para elaborar su teoría del montaje en el cine.

El hanamichi no escapa a la voraz rapiña de Meyerhold en tanto instrumento para que el espectador olvide él, bien por medios arquitectónicos o bien de puesta en el, bien por medos arquilectónicos o bien de puesta en

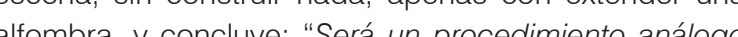
l'camino florido' del teán unós"s6. Años más tar-

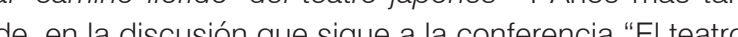

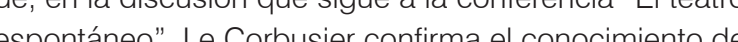
los elementos de la escena del Kabuki: "Creo neeseli que se pueda habler delante de la escena. El teatro iaponés ha utilizado esos artificios"87. Asíf el han como escueto e do esos a las dos partes de Patacio de los Soviets, es el catalizador del deseo de romper con los teatro direccional de la perspectiva con el escer con cajón interior y el elemento aue confirm a voluntar de hacer un lugar de espectáculos al aire libre, un anfiteatro modeno para las grandes manifestaciones de masas del Estado Soviético.

Al sacar el espectáculo a la calle en el Palacio de os Soviets, esta "sala" exterior se configura con un sincretismo entre el teatro direccional y el añadido un sinLa disposición del hanamichi en el eje de simetría sigue la posición más frecuente que se adopta en su traducción europea frente a su situación desplazada hacia la izquierda de la plataforma en las soluciones previas posición exacta respecto al escenario del teatro Kabuki El foro inicial se metamorfosea en un gran auditorio delimitado, frontal y posteriormente, por formas convexas, cuyo centro se vacía con el "foso" que separa la plataforma de la tribuna. Dos niveles de espectáculo formados por la tribuna-hanamichi-plataforma y el nivel del suelo proporcionan múltiples posibilidades de actuación, a la vez que se puede tanto contemplar el espectáculo urcon cos como ser contemplados desde caja escrica y la sala por ambos laterales, y concentra

En el mismo sentido Meyerhold, al contrario de lo que se sucle cre por sulich a constructivismo y nada de la disposición triangular y la simetrí:"La composición tiangur no es el centro del pensamiento en elcampo de apuesta en escena pero es una de as categoŕs cómodas, uno de los elenentos de la puesta en escena que resulta útil par toda un sere de interesantes inenciones pueda el espectedor ver la mayor pate de los puntos de

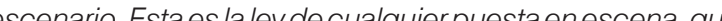
puede obtenerse también con la composición frontal"se. La relación espectador espectáculo es lo que determina finalmente los distintos tipos de arquitectura teatral su distancia y posición relativa. Por otro lado, el edificio leatral ha senido para todo tipo de espectáculos, una de cuyas consecuencias es la mezcla entre auditorio yna de algo que en las apreciaciones del joven Jeannerety en las bases del concurso para el Palacio de los Soviets queda patente. Además a ello se añade, como se ha expuesto, la visibilidad entre los espectadores. Así, con todos estos ingredientes, Le Corbusier propone las dos grandes salas interiores requeridas y una de propina en el exterior En lo que concierne a las salas interiores, asigna cada sala a un tipo de espectáculo. La gran sala es un inmenso auditorio, mientras que la de 6.000 espectadores se disdad. Con estle procediniento Le Corbusier destruye la

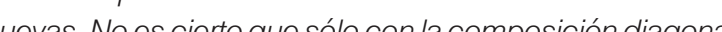
pone como un teatro con caja escénica más tradicional.

86. Meyerhold, Vsevolod: "La composición espacial del espectáculu" (1936). En Meyerhold, Vsevolod: Textos teóricos. Volumen 1. Madrid: Alberto Corazón, 1972, p. 304. "Ce sera une technique analogue au "chemin des fieurs' dans le theâtre japonais". Meyerhold, Vsevolodd: "De quelques questions relatives à l composition spatiale du spectacle" (1936). En Écrits sur let thêâtre. Tome IV, 1936-1940. Laus

87. "Je crois qu'il faut que 'on puisse parler devant la scène. Le thêâtre japonais a usé de ces trucs-lä" Le Corbusier. "Le theâtre spontané" (1948). En Vililiers, André (Press. por): Architiecture et dramaturugie. Op. cit, p. 162

88. Meyerhold, Vsevolod: "La composición espacial del espectáculo" (1936). 0p. cit,, pp. 303-304. "La composition en triangle r'est pas le centre de la est inexact de prétendre que seule la composition en diagonale rend perceptible au spectateur la majoritéé des points. C'est l'objectif de tout jeu de scène, qu peut aussi bien être obtenu avec une composition frontale"." Meyerhold, Vsevolod: "De quelques questions relatives à la composition spatiale du spectacle" (1936). 0p. citt, p. 61
Las influencias de proyectos teatrales soviéticos se dejan notar. El concurso para un teatro de actividades musicales de masas en la ciudad ucraniana de Jarkov, convocado en jullo de 1930 y ganado por Alexander y Viktor Vesnin, tiene mucha más acogida en cuanto al número de proyectos exran mes ye alcanzan riveles de calidad muy sobresalentes. Según De Feo, este concurso marca los Soviets el principio de a decadenci $2^{39}$ Entre lo merosa a luencia de propuestas cancurrentes Von Moos aprecia las semejenzas del Palacio de los Soviets de Le Corbusier sen el teatro de Jarkov (1931) de V Gerasimov y S. Kravets y el proyecto de teatro de Sverdlovik (1030) S. Kravets y el proyecto de teatro de Sverdlovsk (1930)

¿Cuál puede ser el motivo por el que Le Corbusier

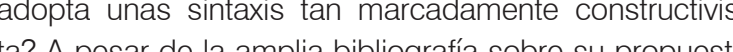
para pl concurso tampoco se ha advertido lo suficiente cerca de la composición del jurado que debe decidir Es evidente que ansioso como está de ponerse a la cabeza de los participantes, no pueda sustraerse a la formación y profesión de las personas que valoran los proyectos y profesión de las person

La profunda frustración que le ocasiona la pérdida en su propio pais de su gran apuesta anterior el Concurso para el Palacio de la SDN de Ginebra, desemboca en la crítica de personas que, aunque con posiciones diversas, hablan un mismo lenguaje, es decir, se dirige a nueve arquitectos ${ }^{91}$ Completamente distinta es la composición el jurado para el Palacio de los Soviets Entre los ocho miembros que lo integran se encuentran el economista Gleb Krzhizhanovsky (presidente), tres arquitectos, M. V. Kriukov, A. Kozelkov y N. P. Zapetlin, los escritores Máximo Gorki y Anatoly Lunacharsky,

89. De Fee, Vittorio: La arquitectura en la URSS 1917-1936 (1963). Op. cit., p. 85. 91. Composicioion del Jurado del concurso para el Palacio de las Naciones, SDN: H. P Berlage ( La Haya), John J.. Burnet (Londres), Carlos Gato (Madrid), Jose

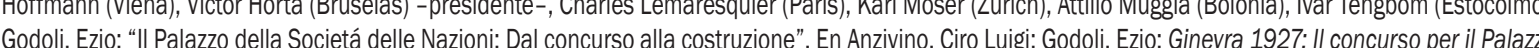
Della Societáa delle Nazioni e il caso Le Corbusier. Firenze: Modulo Editrice, 1979, p. 81.

92. Recogido en De Jong, Cees; Mattie, Erik: Architectural compettions 1, 1792-1949. 0p. cit., p. 285.

93. “" [...] tout simplement parce que son projett à lui était basé sur une analyse approfondie du problème et que la force créatrice de l'architecte n'y était pas entravee dès le principe par l' image arbitraire d'un decor theatral'. En Le Corbusier et Pierre Jeanneret. OEurre complète 1929-34 (1934). Op. cit., p. 7 . 94. Le Corbusier et Pierre Jeanneret OEurre complète 1929-34 (1934). Op. cit, p. 13. 
Algún tiempo después Meyerhold corrobora en "La composición espacial del espectáculo" (1936) las artes que interiorizan el constructivismo: "el constructivismo vive en el campo de la arquitectura y el teatro"s. La heterogenea procedencia profesional de los miembros del jurado puede haber inducido a Le Corbusier a hacer concesiones, no tanto a arquitectos conservadores como a hombres del espectáculo; y aunque esta hipotesis se mantiene en el campo de la conjetua, es raro que su perspicacia pase por allo cuestión lan transcendental. El apoyo del jurado a los proyectos academicistas, en detrimento de la vanguardia arquilectónica, parece deberse a una falla de comprensión arquitectorica, a pesar del enpeno de Le Corbusier quier procedencia y justificar las soluciones adoptadas todo momento permanece, infatigable, al acecho"g8.

sobre criterios de utilidad. Meyerhold expone a renglón (a) ausa del desencuentro. "ni siquier el constructivismo arquitector La capacidad de te

La capacidad de Le Corbusier para apropiarse, sin desvelar el origen, de experiencias culturales y arquitectónicas del entorno en el que quiere convencer, y la gran maestria para explicarlas como un nuevo fruto, revelan a ese enorme camaleôn que, sin renunciar a Guadet ${ }^{9}$ concibe su obra con la astucia de quien juega a caballo ganador, una actitud que reconoce en el comentario (1045): "El resula los Soviels en "L'espace indicible" (1945). "El resultado es esa indiscutible biologia. Ése es no y exterio, unsta toma de posesion del espacio intertodo mono, ilo menda por una voluniad plastica que en

\section{Bibliografía}

A.W.: Frederick Kiesler, Artiste-architecte. Paris: Centre Georges Pompidou, 1996.

Allen Brooks, H. (Ed.): The Le Corbusier archive. Vol. 9. New York-Paris: Garland Publishing-Fondation Le Corbusier, 1982-1984.

Allen Brooks, H.: Le Corbusier 1887-1965 (1987). Milano: Electa, 1993

Capitel, Antón: La arquitectura compuesta por partes. Barcelona: Gustavo Gili 2009.

Cohen, Jean-Louis: Le Corbusier et la mystique de r'URSS. Théories et projets pour Moscou, 1928-1936. Bruxelles-Liège: Pierre Mardaga, 1987. Colquhoun, Alan: Modernidad y tradición clásica (1989). Madrid: Júcar, 1991

Cooke, Catherine; Kazus, Igor: Soviet architectural competitions: 1920s-1930s. London: Phaidon, 1992

Dabrowski. Mardalena y otros: Liubov Popova 1889-1924. Madrid-Munich: Museo Nacional Centro de Arte Reina Sofía-Prestel Verlag. 1991

De Jong, Cees; Mattie, Erik: Architectural competitions 1, 1792-1949. Köln: Taschen, 1994.

.

Frampton, Kenneth: Le Corbusier (1997). Madrid: Akal, 2000.

Gresleri, Giuliano; Zannier, Italo: Viaggio in Oriente. Gli ineditid di Charles-Edouard Jeanneret. Fottografo e scrittore. Venezia-Paris: Marsilio-FLC, 1984

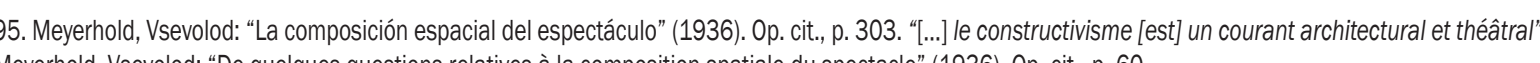

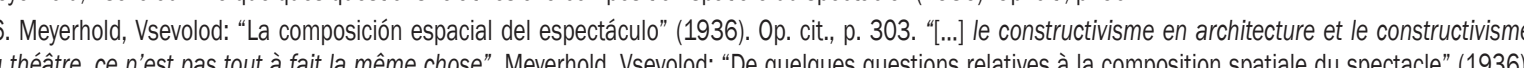
Op. cit, p. 60 .

97. Lucan, Jacques: "Lespace convexe: Le Corbusier et le plan libre". En Lucan, Jacques: Composition non-composition. Architecture et théories, XXXe-XX siècles. Op. cit. 98. Le Corbusier. “El espacio inefable" "("L'espace indicible", 1945. En L'Architecture d'Aujourd"hui, n. hors-série, "Art", 1946, pp. 9-17). En Minerva 02, junio
2006, pp. 6-11, p. 8.
Haan, Hilde; Haagsma, Ids:Architects in competition: internationalarchitectural competitions ofthe last 200 years. London: Thames and Hudson, 1988. Hartnoll, Phyllis: A Concise History of the Theatre (1968). London: Thames and Hudson, 1980.

Hoisington, Sona Stephan: " "Ever Higher': The Evolution of the Project for the Palace of Soviets". Slavic Review, Vol. 62, № 1, Spring 2003, pp. 41-68. Laventiev, Alexander: Varvara Stepanova. A constructivista life. London: Thames and Hudson, 1988.

Le Corbusier: Choix de lettres. Basel-Boston-Berlin: Birkhäuser-Editions d'Architecture, 2002.

e Corbusier: Correspondance. Lettres à sa familie. Tome l: 1900-1925. Paris: Infolio, 2011Etude sur le mouvement d'art décoratiff en Allemagne. La Chaux-de-Fonds: Fondation Le Corbusier, 1912.

Le Corbusier: L'Art decoratif d'aujourd'hui (1925). Paris: Flammarion, 1996.

Le Corbusier: Les voyageses d'Allemagne. Carnets. Paris-Milano: Electa-FLC, English Edition, 1994-2002.

Le Corbusier: Lettres à Charles L'Eplattenier. Paris: Éditions du Linteau, 2007.

Le Corbusier: Hacia una arquitectura (Vers une architecture, 1923). Barcelona: Poseidón, 1978.

Le Corbusier: Le Corbusier et Pierre Jeanneret. OEuvre complète 1929-34 (1934). Zurich: Les Éditions d'Architecture, 1995.

Le Corbusier: Le Corbusier et son atelier rue de Sèvres 35. OEurre complète 1957-1965 (1965). Zurich: Les Éditions d'Architecture, 1995.

Le Corbusier: “Le theâtre spontané" (1948). En Villiers, André (Pres. por): Architecture et dramaturgie. Paris: Flammarion, 1950.

Lucan, Jacques (Ed.): Le Corbusier, une encyclopédie. Paris: Centre Georges Pompidou, 1987.

Lucan, Jacques: Composition, non-composition. Architecture et théories, XIXe-XXe siècles. Vol. 1. Lausanne: Presses polytechniques et universitaires romandes (PPUR), 2009.

Meyerhold, Vsevolod: Textos teóricos. Volumen 1 y 2. Madrid: Alberto Corazón, 1972.

Niessen, Carl: Max Reinhardt und seine Bühnenbildner. Köln: Greven\& Bechtold, 1958.

Pauly, Danièle: Theấtre années 20. La rénovation scénique en France. Paris: Norma 1995.

Pehnt, Wolfgang: La arquitectura expresionista. Barcelona: Gustavo Gili. 1975.

Polieri. lacaues: 50 ans de

Posener, Julius (A cargo de): Hans Poelzig. Scritti e opere (1970). Milano: Franco Angeli Editore, 1978.

Posener, Julius: Hans Poelzig. Sein leben, sein werk. Braunschweig-Wiesbaden: Viewer, 1994.

Quesada, Fernando: La caja mágica. Cuerpo y escena. Barcelona: Fundación Caja de Arquitectos, 2005.

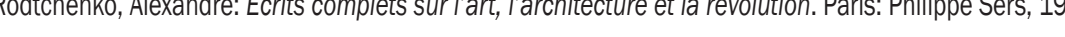

Samonà, Alberto (A cargo de): II Palazzo dei Soviet, 1931-1933. Roma: Officina, 1976.

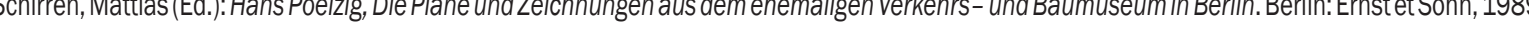
Styan, L. J.: Max Reinhardt. Cambridge-London-New York- New Rochelle- Melbourne-Sydney: Cambridge University Press, 1982. Usandizagá Calparsoro, Miguel: La escena tragícomica. Director Tesis Doctoral: Helio Pinonon, Universitat Politécnica de Catalunna, Departament de

Trevisan, Alexandra, Gonzalez Cubero, Josenna, Veira de Almeida, Pedro (Ed.): Ler Le Corbusier. Porto: CEAA, 2012 (2009).

Von Moos, Stanislaus: Le Corbusier (1968). Barcelona: Lumen, 1994.

Josefina González Cubero, profesorar Titular de Universidad del Departamento de Teoria de la Arquitectura y Proyectos arquiEntre sus intereses de investigación se encuentran la obra de Le Corbusier y la relación entre arquitectura y otras artes. 


\section{Autor imagen y fuente bibliográfica de procedencia}

Información facilitada por los autores de los artículos: página 20, 1 (AA.W.: Concurso de Proyectos 1996. Propuestas de los equipos adjudicatarios. Sevilla: Dirección General de Arquitectura y Vivienda, Junta Andalucía, 1997, portada), 2 (Dirección General de Arquitectura y Vivienda, Junta Andalucía, tríptico); página 22, 3 (Manuel Toledo), 4 (Andrés López); página 23, 5 y 6 (Jesús Granada) 24, 7 (Fernando Alada), 8 (Jesús Granada), 9 (Fernando Alda); páginas 42, 43 y 44, 1 a 3 (El Croquis n53 0MA/Rem Koolhaas. Madrid: El Croquis, 1994, pp. 73 y 78); páginas 46, 47, 4 a 6 (AA.VV: Exposición Universal Sevilla 1992: ideas para una ordenación del recinto, Sevilla: Expo 92, Comisaría General de España, 1986); página 49, 7 (Ceccarelli, P. La construcción de la ciudad soviética. Barcelona: Gustavo Gili, S.A., 1972, figura 26, Anexo ilustraciones, p. XI), 8 (De Michelis, M.; Pasini, E. La Citta`Sovietica 1925-1937, Venecia: Marsilio Editori, 1976, p. 80); páginas 50y 51, 9 y 10 (9. De Michelis, M.; Pasini, E. La Citta 'Sovietica 1925-1937, Venecia: Marsilio Editori, 1976, pp. 81y 180); página 55,1 (Reproducido de Deutches Dokumentation fur Kunstgeschichte, Universitat Marburg, en www.fotomarburg.de); página 56, 2 (Cristina Gastón Guirao); página 57, 3 (Der Scherei nach den Turmhaus. Der IdeenwettbewerbHochhaus am Bahnhof Friedrichstrasse. Berlin 1921/22. Berlín: Argon Verlag GmbH, 1988, p. 39), 4 (Cristina Gastón Guirao en base a Der Scherei nach den Turmhaus. Der IdeenwettbewerbHochhaus am Bahnhof Friedrichstrasse. Berlin 1921/22. Berlín: Argon Verlag GmbH, 1988, p. 38); página 58, 5 (AAVV. Mies in Berlin. (publicado en relación con la exposición del mismo nombre). New York/Berlín: MOMA, 2001, p. 183); página 59, 6 (Reuter, Helmut; Schulte, Birgit. Mies and modern living. Interiors, furniture y photography. Ostfildern: Hatje Canz Verlag, 2008, p. 50, 210 y AAVV. Mies in Berlin. (publicado en relación con la exposición del mismo nombre). New York/Berlín: MOMA, 2001, p.181), 7 (AAVV. Mies in Berlin. (publicado en relación con la exposición del mismo nombre). New York/Berlín: MOMA, 2001, p. 182); página 60, 8 (Cristina Gastón Guirao en base a Der Scherei nach den Turmhaus. Der IdeenwettbewerbHochhaus am Bahnhof Friedrichstrasse. Berlin 1921/22. Berlin: Argon Verlag GmbH, 1988, pp. 45, 105); página 62, 9 y 10 (Reuter, Helmut; Schulte, Birgit. Mies and modern living. Interiors, furniture y photography. Ostfildern: Hatje Canz Verlag, 2008, pp. 230, 252, 258); página 64, 11 (Zevi, Bruno (ed): Erich Mendelsohn. Opera Completa. Turín: Testo \& Immagine, 1997, p. 197), 12 (Der Scherei nach den Turmhaus. Der IdeenwettbewerbHochhaus am Bahnhof Friedrichstrasse. Berlin 1921/22. Berlín: Argon Verlag GmbH, 1988, pp. 181, 184); página 65, 13 (Schulze, Franz. Mies van der Rohe. Una biografía crítica. Madrid: Hermann Blume, 1986 p. 155); página 69, 1 (Inv. Nr. HP 041,005. Architekturmuseum der Technischen Universität Berlin in der Universitätsbibliothek); páginas 70, 72, 73, 74, 2 a 5 (@FLC/Vegap, Sevilla, 2012. @Pierre Jeanneret, Vegap, Sevilla, 2012), 6 (Theaterwissenschaftliche Sammlung Universität zu Köln); página 75, 7 y 8 (Inv. Nr. 2750, Inv. Nr. F 1604. Architekturmuseum der Technischen Universität Berlin in der Universitätsbibliothek); páginas 76 y 80, 9 a 11 (@FLC/Vegap, Sevilla, 2012. @Alexander Rodchenko, Vegap, Sevilla, 2012), 12 (0148126. Tretyakov State Gallery, Moscow); página 82, 13 (Austrian Frederick and Lillian Kiesler Private Foundation Vienna), 14 ((СFLC/Vegap, Sevilla, 2012. @Pierre Jeanneret, Vegap, Sevilla, 2012); página 84, 15 (@FLC/Vegap, Sevilla, 2012. @Pierre Jeanneret, Vegap, Sevilla, 2012), 16 (X1119.3a-c. Colletion of Brooklyn Museum, New York); página, 85, 17 (Cabinet Ejzenstejn, Moscow (Hayuнoмемориальный кабинет-музей С. М. Эйзенштейна), 18 (Imagen de dominio público. http://commons.wikimedia.org/wiki/File:Sadanji_Ichikawa_Il_and_Sergei_Eisenstein. jpg); páginas 92 y 94, 1 y 2 (Roth, Alfred: La nouvelle architecture presentée en 20 exemples, Die neue architektur dargestellt an 20 Beispielen, The new architecture presented in 20 examples. 1930-1940. Zúrich: Verlag für Architektur Artemis, 1975, pp. 131, 132); página 94, 3 (Architettura, № XVI, Fasc. IX. Milán: septiembre de 1938, p. 574); página 96, 4 (Roth, Alfred: La nouvelle architecture presentée en 20 exemples, Die neue architektur dargestellt an 20 Beispielen, The new architecture presented in 20 examples. 1930-1940. Zúrich: Verlag für Architektur Artemis, 1975, p. 136), 5 (Fondazione Giuseppe Terragni; reproducida en Curtis, William J. R.: La arquitectura moderna desde 1900. Londres: Phaidon, 2006, p. 366); página 97, 6 (International Institute of Social History, Amsterdam; reproducida en Molema, Jan: Jan Duiker. Barcelona: Gustavo Gili, 1996, p. 85.), 7 (Emilio Cachorro Fernández); página 98, 8 y 10 (Roth, Alfred: La nouvelle architecture presentée en 20 exemples, Die neue architektur dargestellt an 20 Beispielen, The new architecture presented in 20 examples. 1930-1940. Zúrich: Verlag für Architektur Artemis, 1975, p. 137), 9 (AA.W.: Iǵnazio Gardella, 1905-1999. Arquitectura a través de un siǵlo. Catálogo de la exposición. Madrid: Ministerio de Fomento, 1999, p. 13); página 100, 11 y 12; página 102, 13 (Maffioletti, Serena: BBPR arquitectura. Banfi, Belgiojoso, Peressutti, Rogers. Sevilla: COAAO, 1996, pp. 47, 56, 67), 14 (Musée du Petit Palais, Ginebra; reproducida en Stoichita, Victor l.: Ver y no ver. Madrid: Siruela, 2005, p. 38); página 108, 1 (Arquitectura. Agosto 1969, № 128. Madrid: Colegio Oficial de Arquitectos de Madrid, 1969. Primera página; Nueva forma: arquitectura, urbanismo, diseño, ambientes, arte. "Concurso Universidad Autónoma de Madrid". Septiembre 1969. № 44. Madrid, 1969. Portada; Nueva forma: arquitectura, urbanismo, diseño, ambientes, arte. "Concurso Universidades Autónomas de Bilbao y Barcelona". Enero 1970. № 48. Madrid, 1970. Portada); página 110, 2 (Nueva forma. Septiembre 1969. № 44. cit. p. 5); página 111, 3 y 4 (Arquitectura. Agosto 1969, № 128. cit. pp. 33 y 35); páginas 112 y 113, 5 a 9 (Nueva forma. Septiembre 1969. № 44. cit. pp. 33, 30, 81, 18, 24); páginas 114, 115 y 116, 10 a 14 (Nueva forma. Enero 1970. № 48. cit. pp. 23, 20, 56, 69, 96), 15 y 16 (Cuadernos de arquitectura. Primer trimestre 1970. № 75. Barcelona: Colegio Oficial de Arquitectos de Catalunya, 1970. pp. 58 y 66); página 117, 17 (Nueva forma. Enero 1970. № 48. cit. p. 31); página 118, 18 (Nueva forma. Septiembre $1969 . N^{\circ} 44$. cit. p. 4; Temas de arquitectura y urbanismo. Septiembre 1969. № 123. Madrid-Barcelona, 1969. p. 5); página 123, 1 y 2 (Bucci, Ângelo; Puntoni, Álvaro; Vilela, José Oswaldo. Foto: Nelson Kon.); página 126, 3 (Parada, Sérgio Roberto. Projeto cedido por Rodrigo Biavati), 4 (Gorgati, Vinicius; Franco, Fernando de Mello; Moreira, Marta; Braga, Milton. Projeto cedido pelos autores), 5 (Rodrigues, Sidney Meleiros; Saraiva, Pedro Paulo de Melo; Rosemberg, Marcelo; Vaisman, Jacobina; Lobo, Marcos Toledo; Nunes, Ronaldo Soares.Projeto cedido pelos autores); páginas 128, 129, 6 a 12 (Bucci, Ângelo; Puntoni, Álvaro; Vilela, José Oswaldo. Projeto cedido pelos autores); página 131, 13 y 14 (Bucci, Ângelo; Puntoni, Álvaro; Vilela, José Oswaldo. Foto: Nelson Kon); página 136, 1 (Fromonot, Françoise. En Jørn Utzon, architetto della Sydney Opera House. Milano: Electa, Documenti di Architettura, 1998. pp. 15), 2 (Piano, Renzo. En Giornale di Bordo. Florencia: Passigli Editori, 1997, pag 38); página 138, 3 (Mikami, Yuzo. En Utzon's sphere. Sydney Opera House. How it was designed and built.Tokio: Ed. Shoku Kusha, 2001), 4 (Silver, Nathan, The making of Beuabourg, A building biography of the Centre Pomidou, Paris. Cambridge, Massachussets: Ed The MIT press, 1994); página 139, 5 (Piano, Renzo, Renzo Piano Building Workshop, complete works. Londres: Phaidon Press Limited, 1993. vol 1. pp. 54), 6 (Weston, Richard: Utzon : inspiration, vision, architecture. Hellerup (Dinamarca): Editorial Blondal, , 2002. pp. 117); página 140, 7 (Weston, Richard: Utzon: inspiration, vision, architecture. Hellerup (Dinamarca): Editorial Blondal, , 2002. pp 116), 8 (Fromonot, Françoise. En Jørn Utzon, architetto della Sydney Opera House. Milano: Electa, Documenti di Architettura, 1998. pp 64), 9 (Ferrer, Jaime, Jørn Utzon. Obras y proyectos. Barcelona: Editorial. Gustavo Gili, 2008. pp. 159); página 10, (Rice, Peter: "La structure métallique". En Architecture d'aujourd'hui. Febrero 1982, n 219. pp. 62. París: Editorial Groupe Expansion,1930), 11 (Piano, Renzo, Renzo Piano Building Workshop, complete works. Londres: Phaidon Press Limited, 1993. vol 1. pp. 45); página 142, 12 (Architectural Design', vol 47 nº 2, febrero 1977); página 144, 13 (Weston, Richard: Utzon : inspiration, vision, architecture. Hellerup (Dinamarca): Editorial Blondal, 2002); página 148, 1 (AA.W.: Arquitectura Viva. "Obras singulares". 1998, № 62. Madrid: Arquitectura Viva. 1998. pp. 78-81); página 149, 2 y 3 (AA.W.: Concurso 2G competition: Parque de la Laguna de Venecia = Venice Laggon Park. Barcelona: Gustavo Gill, 2008. pp. 81, 13); página 150, 4 (Jacopo de 'Barbari, [Web en linea]. [Consulta: 04-10-2012]), 5 (Zumthor, Peter: Atmósferas: entornos arquitectónicos, las cosas a mi alrededor. Barcelona: Gustavo Gili, 2006. p. 6); página 151, 6 (David Chipperfield 2006-2009. El Croquis. № 120. Madrid: El Croquis. 2008. p. 66); páginas 152, 153,7 a 9 (AA.W.: Carlos Ferrater. Works and Projects 1980-2000. Barcelona: Actar, 1998); páginas 154, 155, 10 a 12 (Enric Miralles + Benedetta Tagliabue: 1995-2000. El Croquis. № 72. Madrid: El Croquis. 2000. pp. 132-141); página 156, 13 a 15 (David Chipperfield 2006-2009. El Croquis. № 120. Madrid: El Croquis. 2008. pp. 64, 67, 66); página 157, 16,17 (Luca Nicolao Photography, [Web en linea]. [Consulta: 19-9-2012]), 18 (David Chipperfield 2006-2009. El Croquis. № 120. Madrid: El Croquis. 2006. p. 66); páginas 162, 164, 167, 169, 170, 1 a 6 (๑ F.L.C. /VEGAP, Sevilla, 2012. OPierre Jeanneret, VEGAP, Sevilla, 2012); página 176, 1 (García Mercadal y S. Giedion. Congresos internacionales de arquitectura moderna. AC. №5. Primer Trimestre de 1935. Barcelona. GATEPAC.1932 p.12); páginas 178, 179, 2 a 4 (AC. №11. Tercer trimestre de 1933. Barcelona. GATEPAC. 1933. pp. 37, 34, 35); página 180, 5 (Le Corbusier et Pierre Jeanneret : Ouvre Complète 1910-1929. Zurich : Les Édtions D’Architecture. 1964 p.65), 6 (AC. NN11. Tercer trimestre de 1933. Barcelona. GATEPAC. 1933. p. 34), 7 (Le Corbusier et Pierre Jeanneret: Ouvre Complète 1910-1929. Zurich : Les Édtions D’Architecture. 1964 p.65), 8 (L'Habitation minimun. Frankfurt. 1929. Zaragoza: Edición facsimil Colegio de Arquitectos de Aragón.1997.p.26), páginas 181, 182 (AC. №11. Tercer trimestre de 1933. Barcelona. GATEPAC. 1933. p. 37, 1); páginas 189, 190, 1 y 2 (Cássia de Souza Mota); página 191, 3 (Boero, Dolores; Castellote, Ana Maria; Puglisi, Jose Agustin. Fonte: http://www.uia-architectes.org/texte/ england/Celebration/SPrix/Reglll/Frames.html), 4 (Fernandez, Alberto; Guerrero, Camilo; Javiera, Paulina; Sanchez Recio, José Manuel. Fonte: http://www.celebcities2.org/); página 192, 5 y 6 (Nikita, Sergienko. Fonte: http://www.celebcities3.org/wc/content/18/pdf/054FECC2-C9DC-410A-A044-CB2A0BBD25EB.pdf) 